 or referenced by the media as validated information. \\ Multistability Analysis of State-Dependent Switched Hopfield Neural Networks With The Gaussian- Wavelet-Type Activation Function*
}

Yang Liu

Shandong University of Science and Technology

Zhen Wang ( $\square$ wangzhen_sd@126.com )

Shandong University of Science and Technology https://orcid.org/0000-0001-7188-5828

\section{Research Article}

Keywords: Multistability, State-dependent switching, Gaussian-wavelet-type activation functions, Equilibrium points, Hopfield neural networks.

Posted Date: August 31st, 2021

DOI: https://doi.org/10.21203/rs.3.rs-831388/v1

License: (c) (i) This work is licensed under a Creative Commons Attribution 4.0 International License.

Read Full License

Version of Record: A version of this preprint was published at Mathematics and Computers in Simulation on February 1st, 2022. See the published version at https://doi.org/10.1016/j.matcom.2022.01.021. 


\title{
Multistability analysis of state-dependent switched Hopfield neural networks with the Gaussian-wavelet-type activation function *
}

\author{
Yang Liu, †'Zhen Wang \\ College of Mathematics and Systems Science, \\ Shandong University of Science and Technology, Qingdao 266590, China
}

\begin{abstract}
This paper studies the multistability of state-dependent switched Hopfield neural networks (SSHNNs) with the Gaussian-wavelet-type activation function. The coexistence and stability of multiple equilibria of SSHNNs are proved. By using Brouwer's fixed point theorem, it is obtained that the SSHNNs can have at least $7^{n}$ or $6^{n}$ equilibria under a specified set of conditions. By using the strictly diagonally dominance matrix (SDDM) theorem and Lyapunov stability theorem, $4^{n}$ or $5^{n}$ locally stable (LS) equilibria are obtained, respectively. Compared with the conventional Hopfield neural networks (HNNs) without state-dependent switching or SSHNNs with other kinds of activation functions, SSHNNs with this type of activation functions can have more LS equilibria, which implies that SSHNNs with Gaussianwavelet-type activation functions can have even larger storage capacity and would be more dominant in associative memory application. Last, some simulation results are given to verify the correctness of the theoretical results.
\end{abstract}

Keywords: Multistability; State-dependent switching; Gaussian-wavelet-type activation functions; Equilibrium points; Hopfield neural networks.

\section{Introduction}

In recent decades, neural networks (NNs) have been widely concerned due to their widespread applications in different fields [1]-[7]. As is known to us all, unique stable equilibrium point is necessary in some applications and it is a basic condition, but for some other applications, such as pattern recognition, associative memories and image processing, multiple equilibria are needed. The existence and stability/instability of multiple equilibrium points are described in multistability of NNs, including the total number of equilibria and the attraction basins of the LS equilibria [8]. In associative memory, addressable memory is stored as a LS equilibrium point. When a randomly given initial condition converges to a stable equilibrium point, we call the process of associative memory completes. So, if a NN can possess more multiple LS equilibria, it means that it has a larger storage capacity. Therefore, many researchers have been attracted to study the multistability. Till now, various kinds of NN models have been proposed in the multistability analysis. To be specific, the Hopfield NNs [9], the delayed NNs [10], the competitive NNs [11], the BAM NNs [12], the Cohen-Grossberg NNs [13], the stochastic delayed NNs [14], the

${ }^{*}$ This work was supported by the NSFC under Grants 62173214, 61973199 and Shandong University of Science and Technology Research Fund (2018 TDJH101).

${ }^{\dagger}$ Corresponding author: wangzhen_sd@126.com 
delayed impulsive NNs [15]-[17] and the discrete-time NNs [18]-[22], have been covered in the study of the multistability.

As we all know, the properties (e.g., the continuity and the monotonicity) of activation functions play an important role in the multistability analysis of NNs, which can determine the number of total equilibria as well as LS equilibria. At first, multistability of NNs with the monotonically nondecreasing functions are usually used to study. For example, the sigmoidal function was used in [23], which is a nonlinear function and the saturated function was used in [24], which is a piecewise linear function. Then, compared with the monotonic activation functions, it was found that the nonmonotonic activation functions could have more equilibria, which makes the nonmonotonic activation function popular in multistability analysis. In [25], the Mexican-hat-type function was designed, which is continuous but nonmonotonic and NNs with this type of activation function could have $3^{n}$ equilibria, $2^{n}$ of which are LS. Inspired by this, the authors in [26] designed a kind of discontinuous piecewise linear function, which is modified from the Mexican-hat-type function and is discontinuous. It was shown that NNs with the proposed discontinuous activation function could produce $3^{n} \mathrm{LS}$ equilibria in total $5^{n}$ equilibria. It is clear that the number of LS equilibria was increased compared with [25], which implies the storage capacity is expanded. Besides, the Gaussian activation function [27], which is a kind of nonlinear function, was used in multistability analysis of NNs. Also, multistability for continuous-time NNs with some other activation functions were discussed in [28]-[32] and some improved results were obtained.

Most recently, a NN model named the state-dependent switched Hopfield neural networks (SSHNNs) was proposed and the multistability of SSHNNs with the sigmoidal function [33], the saturated function [34] and the Mexican-hat-type function [35] were respectively studied. Moreover, it has been proven that under some conditions, the SSHNNs can have more LS equilibria compared with the HNNs with the same type of activation functions [33]-[35]. So we may get a conclusion that, the SSHNNs model may have much richer dynamical behaviors than HNNs model even if they use the same type of activation function. In [36], a kind of activation function named Gaussian-wavelet-type activation function was proposed and HNNs with this type of activation function can have more total equilibria and stable equilibria than HNN with the sigmoidal function, the saturated function or the Mexican-hat-type function. Inspired by the above discussions, in this paper, we are going to study the multistability of SSHNNs with Gaussian-wavelet-type activation function, which is defined by

$$
f_{i}(\xi)= \begin{cases}u, & -\infty<\xi<-k \\ l \xi+c, & -k \leq \xi \leq 0 \\ -l \xi+c, & 0<\xi \leq k \\ \bar{l} \xi+\bar{c}, & k<\xi \leq d \\ v, & d<\xi<+\infty\end{cases}
$$

where $l, \bar{l}, k, u, v, d, c, \bar{c}$ are constants with $l>0, \bar{l}>0, d>k>0, u=f_{i}(k)=f_{i}(-k)$ and $v \geq c, i=$ $1,2, \ldots, n$. Fig. 1 displays the graph of the Gaussian-wavelet-type activation function (1). 


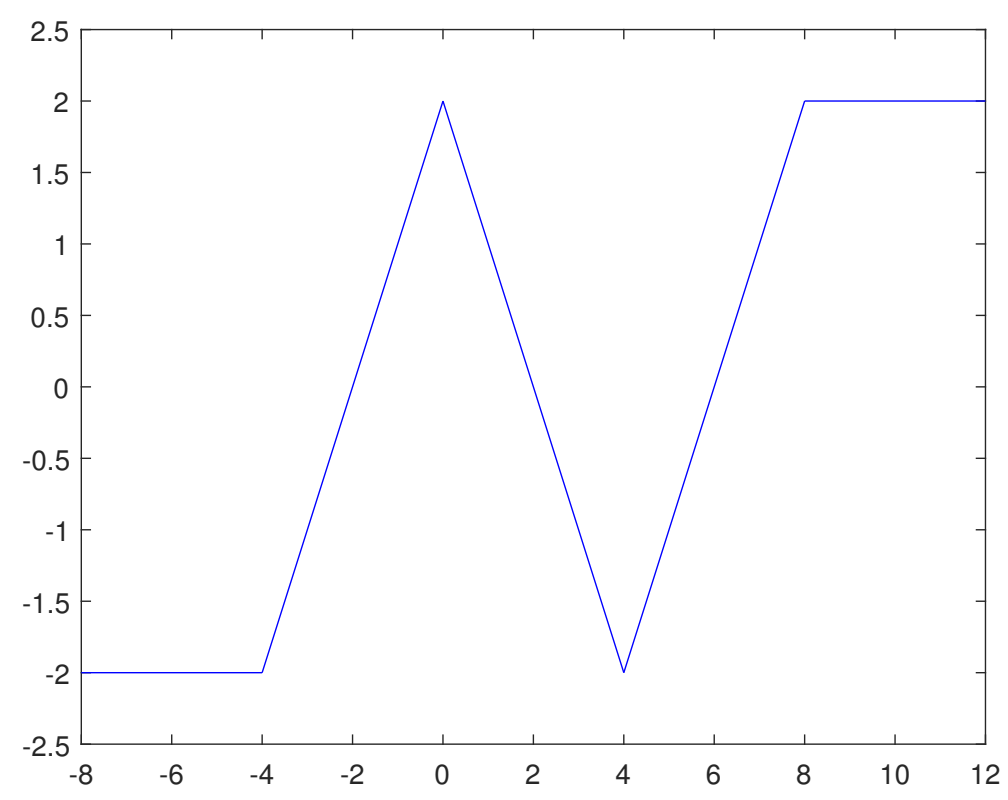

Fig. 1. The Gaussian-wavelet-type activation function defined in (1).

In this paper, we're going to focus on multistability and instability of SSHNNs with the Gaussianwavelet-type activation function (1). The main contributions can be summarized as follows: 1) We obtain some sufficient conditions such that the $n$-neuron SSHNNs has $6^{n}$ or $7^{n}$ equilibrium points in total depending on the relationship between the switching threshold $T$ and $k, d$. 2) The positively invariant set of SSHNNs is clearly identified to better study the stability of multiple equilibria. 3) $4^{n}$ or $5^{n}$ LS equilibria in total $6^{n}$ or $7^{n}$ equilibria can be identified by using the property of strictly diagonally dominance matrix and the Lyapunov stability theorem. 4) Compared with the existing results, it can be concluded that NNs with state-dependent switching can have even more stable equilibria, which implies that SSHNNs can have even larger storage capacity.

Notation. $\mathbb{R}^{n}$ denotes the $n$-dimensional Euclidean space and $\rho=\left(\rho_{1}, \rho_{2}, \ldots, \rho_{n}\right)^{T} \in \mathbb{R}^{n}$ denotes a $n$-dimensional vector. Denote $K[E]$ as the closure of the convex hull of $E$ for a given set $E \in \mathbb{R}^{n}$.

\section{Preliminaries}

\section{$2.1 \quad$ Model description}

In this paper, we consider a class of SSHNNs described by

$$
\frac{d \rho_{i}(t)}{d t}=-b_{i}\left(\rho_{i}(t)\right) \rho_{i}(t)+\sum_{j=1}^{n} a_{i j}\left(\rho_{i}(t)\right) f_{j}\left(\rho_{j}(t)\right)+J_{i}, i=1,2, \ldots, n, \quad t \geq 0
$$

where $\rho(t)=\left(\rho_{1}(t), \rho_{2}(t), \ldots, \rho_{n}(t)\right)^{T} \in \mathbb{R}^{n}$ denotes the state vector; $B(\rho)=\operatorname{diag}\left(b_{1}\left(\rho_{1}\right), b_{2}\left(\rho_{2}\right), \ldots, b_{n}\left(\rho_{n}\right)\right)$ represents the neuron self-feedback; $A(\rho)=\left(a_{i j}\left(\rho_{i}\right)\right)_{n \times n}$ represents the connection weight matrix; $f(\rho)=$ $\left(f_{1}\left(\rho_{1}\right), f_{2}\left(\rho_{2}\right), \ldots, f_{n}\left(\rho_{n}\right)\right)^{T}$ denotes the activation function and $J=\left(J_{1}, J_{2}, \ldots, J_{n}\right)^{T}$ denotes the input vector. For $i, j=1,2, \ldots, n$, the components of $B(\rho)$ and $A(\rho)$ satisfy the following conditions:

$$
b_{i}\left(\rho_{i}(t)\right)= \begin{cases}b_{i}^{\prime}, & \left|\rho_{i}(t)\right| \leq T \\ b_{i}^{\prime \prime}, & \left|\rho_{i}(t)\right|>T\end{cases}
$$


and

$$
a_{i j}\left(\rho_{i}(t)\right)= \begin{cases}a_{i j}^{\prime}, & \left|\rho_{i}(t)\right| \leq T \\ a_{i j}^{\prime \prime}, & \left|\rho_{i}(t)\right|>T\end{cases}
$$

where $T>0$ denotes the switching threshold, $b_{i}^{\prime}, b_{i}^{\prime \prime}$ and $a_{i j}^{\prime}, a_{i j}^{\prime \prime}$ are all constants with $b_{i}^{\prime}>0, b_{i}^{\prime \prime}>0$. It is clear that the system (2) switches between two different groups of parameters depending on the location of the states.

\subsection{Definitions and lemmas}

Next, several lemmas and definitions will be given. First of all, denote $K[f(\rho)]=\left(K\left[f_{1}\left(\rho_{1}\right)\right], K\left[f_{2}\left(\rho_{2}\right)\right]\right.$ $\left., \ldots, K\left[f_{n}\left(\rho_{n}\right)\right]\right)^{T}$, where $K\left[f_{i}\left(\rho_{i}\right)\right]=\left[f_{i}\left(\rho_{i}^{-}\right), f_{i}\left(\rho_{i}^{+}\right)\right]$. Let $\check{b}_{i}=\min \left\{b_{i}^{\prime}, b_{i}^{\prime \prime}\right\}, \hat{b}_{i}=\max \left\{b_{i}^{\prime}, b_{i}^{\prime \prime}\right\}, \check{a}_{i j}=$ $\min \left\{a_{i j}^{\prime}, a_{i j}^{\prime \prime}\right\}$ and $\hat{a}_{i j}=\max \left\{a_{i j}^{\prime}, a_{i j}^{\prime \prime}\right\}$, denote $K[B(\rho)]=\operatorname{diag}\left(K\left[b_{1}\left(\rho_{1}\right)\right], K\left[b_{2}\left(\rho_{2}\right)\right], \ldots, K\left[b_{n}\left(\rho_{n}\right)\right]\right), K[A(\rho)]$ $=\left(K\left[a_{i j}\left(\rho_{i}\right)\right]\right)_{n \times n}$ for $i, j=1,2, \ldots, n$, where

$$
K\left[b_{i}\left(\rho_{i}(t)\right)\right]=\left\{\begin{array}{ll}
b_{i}^{\prime}, & \left|\rho_{i}(t)\right|<T \\
b_{i}^{\prime \prime}, & \left|\rho_{i}(t)\right|>T \\
{\left[\breve{b}_{i}, \hat{b}_{i}\right],} & \left|\rho_{i}(t)\right|=T
\end{array} \quad \text { and } \quad K\left[a_{i j}\left(\rho_{i}(t)\right)\right]= \begin{cases}a_{i j}^{\prime}, & \left|\rho_{i}(t)\right|<T \\
a_{i j}^{\prime \prime}, & \left|\rho_{i}(t)\right|>T \\
{\left[\check{a}_{i j}, \hat{a}_{i j}\right],} & \left|\rho_{i}(t)\right|=T\end{cases}\right.
$$

Definition 1 [34]. For a vector $\rho^{*} \in \mathbb{R}^{n}$, if it satisfies that

$$
0 \in-K\left[B\left(\rho^{*}\right)\right] \rho^{*}+K\left[A\left(\rho^{*}\right)\right] f\left(\rho^{*}\right)+J .
$$

then, $\rho^{*}$ is an equilibrium point of $\operatorname{SSHNN}(2)$,

Definition 2 [26]. For a real matrix $A=\left(a_{i j}\right)_{n \times n}$, if it satisfies that

$$
\left|a_{i i}\right|>\sum_{j=1, j \neq i}^{n}\left|a_{i j}\right|, i=1,2, \ldots, n \text {. }
$$

Then, $A$ is called a SDDM.

Lemma 1 [37]. If $A=\left(a_{i j}\right)_{n \times n}$ is a SDDM, then the matrix $A$ can have the following properties:

1) For $i=1,2, \ldots, n$, if $a_{i i}>(<) 0$, then every eigenvalue of $A$ has the positive (negative) real part;

2) Denote $K_{1}=\left\{i \mid a_{i i}<0, i=1,2, \ldots, n\right\}$ and $K_{2}=\left\{i \mid a_{i i}>0, i=1,2, \ldots, n\right\}$. If the number of elements in $K_{1}$ is $n_{1}$ and the number of elements in $K_{2}$ is $n_{2}, n_{1}+n_{2}=n$, then $n_{1}$ eigenvalues of $A$ have the negative real part and $n_{2}$ eigenvalues of $A$ have the positive real part.

\section{Main results}

In this section, we will study the dynamical behaviors of SSHNNs (2) with the Gaussian-wavelet-type activation function (1). According to the properties of the above activation function, we will discuss from the following three cases: when switching threshold $T$ is located in $(0, k),(k, d)$ and $(d,+\infty)$, respectively.

First, consider the case $0<T<k$, i.e., when the switching threshold $T$ is located in $(0, k)$, the coexistence and stability of equilibria will be analysed. 


\subsection{Coexistence of multiple equilibrium points}

First, the coexistence of equilibria will be studied. Denote

$$
\begin{aligned}
(-\infty,-k) & =(-\infty,-k)^{1} \times(-k,-T)^{0} \times(-T, 0)^{0} \times(0, T)^{0} \times(T, k)^{0} \times(k, d)^{0} \times(d,+\infty)^{0} \\
(-k,-T) & =(-\infty,-k)^{0} \times(-k,-T)^{1} \times(-T, 0)^{0} \times(0, T)^{0} \times(T, k)^{0} \times(k, d)^{0} \times(d,+\infty)^{0} \\
(-T, 0) & =(-\infty,-k)^{0} \times(-k,-T)^{0} \times(-T, 0)^{1} \times(0, T)^{0} \times(T, k)^{0} \times(k, d)^{0} \times(d,+\infty)^{0} \\
(0, T) & =(-\infty,-k)^{0} \times(-k,-T)^{0} \times(-T, 0)^{0} \times(0, T)^{1} \times(T, k)^{0} \times(k, d)^{0} \times(d,+\infty)^{0} \\
(T, k) & =(-\infty,-k)^{0} \times(-k,-T)^{0} \times(-T, 0)^{0} \times(0, T)^{0} \times(T, k)^{1} \times(k, d)^{0} \times(d,+\infty)^{0} \\
(k, d) & =(-\infty,-k)^{0} \times(-k,-T)^{0} \times(-T, 0)^{0} \times(0, T)^{0} \times(T, k)^{0} \times(k, d)^{1} \times(d,+\infty)^{0} \\
(d,+\infty) & =(-\infty,-k)^{0} \times(-k,-T)^{0} \times(-T, 0)^{0} \times(0, T)^{0} \times(T, k)^{0} \times(k, d)^{0} \times(d,+\infty)^{1}
\end{aligned}
$$

and denote a set $\Omega_{n}$ as

$$
\begin{aligned}
\Omega_{n}= & \left\{\prod_{i=1}^{n}(-\infty,-k)^{\delta_{1}^{(i)}} \times[-k,-T]^{\delta_{2}^{(i)}} \times(-T, 0)^{\delta_{3}^{(i)}} \times[0, T]^{\delta_{4}^{(i)}} \times(T, k)^{\delta_{5}^{(i)}} \times(k, d)^{\delta_{6}^{(i)}} \times(d,+\infty)^{\delta_{7}^{(i)}},\left(\delta_{1}^{(i)},\right.\right. \\
& \left.\delta_{2}^{(i)}, \delta_{3}^{(i)}, \delta_{4}^{(i)}, \delta_{5}^{(i)}, \delta_{6}^{(i)}, \delta_{7}^{(i)}\right)=(1,0,0,0,0,0,0) \text { or }(0,1,0,0,0,0,0) \text { or }(0,0,1,0,0,0,0) \text { or }(0,0,0,1, \\
& 0,0,0) \text { or }(0,0,0,0,1,0,0) \text { or }(0,0,0,0,0,1,0) \text { or }(0,0,0,0,0,0,1)\} .
\end{aligned}
$$

It is shown that the set $\Omega_{n}$ have $7^{n}$ regions in total. Assume that $\varepsilon$ is small enough and $0<\varepsilon \ll$ $\min _{1 \leq i \leq n}\left\{1 / k, b_{i}^{\prime \prime} /\left(\sum_{j=1}^{n}\left|a_{i j}^{\prime \prime}\right| \mu+\left|J_{i}\right|\right)\right\}$, where $\mu=\max \{|u|,|v|\}$. Then, denote a set

$$
\begin{aligned}
\Omega_{\varepsilon}= & \left\{\prod_{i=1}^{n}[-1 / \varepsilon,-k-\varepsilon]^{\delta_{1}^{(i)}} \times[-k+\varepsilon,-T-\varepsilon]^{\delta_{2}^{(i)}} \times[-T+\varepsilon,-\varepsilon]^{\delta_{3}^{(i)}} \times[\varepsilon, T-\varepsilon]^{\delta_{4}^{(i)}} \times[T+\varepsilon, k-\varepsilon]^{\delta_{5}^{(i)}} \times[k\right. \\
& +\varepsilon, d-\varepsilon]_{6}^{\delta_{6}^{(i)}} \times[d+\varepsilon, 1 / \varepsilon]_{7}^{\delta_{7}^{(i)}},\left(\delta_{1}^{(i)}, \delta_{2}^{(i)}, \delta_{3}^{(i)}, \delta_{4}^{(i)}, \delta_{5}^{(i)}, \delta_{6}^{(i)}, \delta_{7}^{(i)}\right)=(1,0,0,0,0,0,0) \text { or }(0,1,0,0,0,0,0) \\
& \text { or }(0,0,1,0,0,0,0) \text { or }(0,0,0,1,0,0,0) \text { or }(0,0,0,0,1,0,0) \text { or }(0,0,0,0,0,1,0) \text { or }(0,0,0,0,0,0,1), i \\
& =1,2, \ldots, n\} .
\end{aligned}
$$

It is shown that for any set $\Omega(\varrho) \in \Omega_{\varepsilon}, \varrho \in\left\{1,2, \ldots, 7^{n}\right\}$, then $\Omega(\varrho)$ is closed and bounded.

Theorem 1 For SSHNN (2) with activation function (1), if the following conditions hold for $i=1,2, \ldots, n$ :

$$
\begin{gathered}
b_{i}^{\prime \prime} k+a_{i i}^{\prime \prime} u+\sum_{j \neq i, j=1}^{n} \max \left\{a_{i j}^{\prime \prime} u, a_{i j}^{\prime \prime} v\right\}+J_{i}<0, \\
-b_{i}^{\prime \prime} T+a_{i i}^{\prime \prime} f_{i}(T)+\sum_{j \neq i, j=1}^{n} \min \left\{a_{i j}^{\prime \prime} u, a_{i j}^{\prime \prime} v\right\}+J_{i}>0, \\
-b_{i}^{\prime} T+a_{i i}^{\prime} f_{i}(T)+\sum_{j \neq i, j=1}^{n} \min \left\{a_{i j}^{\prime} u, a_{i j}^{\prime} v\right\}+J_{i}>0, \\
a_{i i}^{\prime} c+\sum_{j \neq i, j=1}^{n} \max \left\{a_{i j}^{\prime} u, a_{i j}^{\prime} v\right\}+J_{i}<0, \\
-b_{i}^{\prime \prime} d+a_{i i}^{\prime \prime} v+\sum_{j \neq i, j=1}^{n} \min \left\{a_{i j}^{\prime \prime} u, a_{i j}^{\prime \prime} v\right\}+J_{i}>0,
\end{gathered}
$$

then $\operatorname{SSHNN}$ (2) can have at least $7^{n}$ equilibria in $\Omega_{\varepsilon}$. 
Before proving Theorem 1, we first raise a proposition to determine the relationship between parameters of the system to make the proof of Theorem 1 more accessible.

Proposition 1 From conditions (5)-(9), we can obtain that $a_{i i}^{\prime \prime}>0, a_{i i}^{\prime}<0,-b_{i}^{\prime \prime}+a_{i i}^{\prime \prime} l>0,-b_{i}^{\prime \prime}+a_{i i}^{\prime \prime} \bar{l}>0$, $-b_{i}^{\prime}-a_{i i}^{\prime} l>0,-b_{i}^{\prime \prime}-a_{i i}^{\prime \prime} l<0$ and $-b_{i}^{\prime}+a_{i i}^{\prime} l<0$, for $i=1,2, \ldots, n$.

Proof: From $(6),(7)$ and $f_{i}(T)=f_{i}(-T), T>-T$, we get that

$$
\begin{aligned}
& b_{i}^{\prime \prime} T+a_{i i}^{\prime \prime} f_{i}(-T)+\sum_{j \neq i, j=1}^{n} \min \left\{a_{i j}^{\prime \prime} u, a_{i j}^{\prime \prime} v\right\}+J_{i}>0, \\
& b_{i}^{\prime} T+a_{i i}^{\prime} f_{i}(-T)+\sum_{j \neq i, j=1}^{n} \min \left\{a_{i j}^{\prime} u, a_{i j}^{\prime} v\right\}+J_{i}>0 .
\end{aligned}
$$

Owing to (5) and (10), we have

$$
b_{i}^{\prime \prime} T+a_{i i}^{\prime \prime} f_{i}(-T)>b_{i}^{\prime \prime} k+a_{i i}^{\prime \prime} u .
$$

That is,

$$
0<b_{i}^{\prime \prime}(k-T)<a_{i i}^{\prime \prime}\left(f_{i}(-T)-u\right) .
$$

It implies $a_{i i}^{\prime \prime}>0$ owing to $f_{i}(-T)>u$, and $-b_{i}^{\prime \prime}+a_{i i}^{\prime \prime} l>0$ owing to $u=f_{i}(-k)$.

From (5) and $k>-k, f_{i}(k)=f_{i}(-k)$, we get that

$$
-b_{i}^{\prime \prime} k+a_{i i}^{\prime \prime} u+\sum_{j=1, j \neq i}^{n} \max \left\{a_{i j}^{\prime \prime} u, a_{i j}^{\prime \prime} v\right\}+J_{i}<0 .
$$

Similarly, owing to (9) and (12), we have

$$
-b_{i}^{\prime \prime} d+a_{i i}^{\prime \prime} v>-b_{i}^{\prime \prime} k+a_{i i}^{\prime \prime} u
$$

So we can get $-b_{i}^{\prime \prime}+a_{i i}^{\prime \prime} \bar{l}>0$. Then, due to $(7),(8)$ and $f_{i}(0)=c$, we obtain that

$$
-b_{i}^{\prime} T+a_{i i}^{\prime} f_{i}(T)>a_{i i}^{\prime} f_{i}(0)
$$

That is,

$$
-b_{i}^{\prime}(T-0)>a_{i i}^{\prime}\left(f_{i}(0)-f_{i}(T)\right),
$$

which implies $a_{i i}^{\prime}<0,-b_{i}^{\prime}-a_{i i}^{\prime} l>0$. Besides, because $a_{i i}^{\prime \prime}>0, a_{i i}^{\prime}<0, b_{i}^{\prime}>0, b_{i}^{\prime \prime}>0$ and $l>0$, we obtain $-b_{i}^{\prime \prime}-a_{i i}^{\prime \prime} l<0,-b_{i}^{\prime}+a_{i i}^{\prime} l<0$. This completes the proof.

Next, by terms of Brouwer's fixed point theorem, Theorem 1 will be proved to confirm the existence of multiple equilibria of SSHNN (2).

Proof of Theorem 1: Pick a region from $\Omega_{\varepsilon}$ arbitrarily and denote it as

$$
\begin{array}{r}
\widetilde{\Omega}_{\varepsilon}=\prod_{i \in N_{1}}[-1 / \varepsilon,-k-\varepsilon] \times \prod_{i \in N_{2}}[-k+\varepsilon,-T-\varepsilon] \times \prod_{i \in N_{3}}[-T+\varepsilon,-\varepsilon] \times \prod_{i \in N_{4}}[\varepsilon, \\
T-\varepsilon] \times \prod_{i \in N_{5}}[T+\varepsilon, k-\varepsilon] \times \prod_{i \in N_{6}}[k+\varepsilon, d-\varepsilon] \times \prod_{i \in N_{7}}[d+\varepsilon, 1 / \varepsilon] \subset \Omega_{\varepsilon}
\end{array}
$$

where $N_{i}$ are the subsets of $\{1,2, \ldots, n\}$ and $N_{i} \cap N_{j}=\emptyset(i \neq j, i, j=1,2,3,4,5,6,7), N_{1} \cup N_{2} \cup N_{3} \cup$ $N_{4} \cup N_{5} \cup N_{6} \cup N_{7}=\{1,2, \ldots, n\}$. Then, we will prove that SSHNN (2) with activation function (1) can have an equilibrium point located in $\widetilde{\Omega}_{\varepsilon}$. 
Pick a point $\left(\rho_{1}, \rho_{2}, \ldots, \rho_{n}\right)^{T} \in \widetilde{\Omega}_{\varepsilon}$ arbitrarily and fix $\rho_{1}, \ldots, \rho_{i-1}, \rho_{i+1}, \ldots, \rho_{n}$ except $\rho_{i}$. Then, define a function

$$
F_{i}(x)=-b_{i}(x) x+a_{i i}(x) f_{i}(x)+\sum_{j=1, j \neq i}^{n} a_{i j}(x) f_{j}\left(\rho_{j}\right)+J_{i}, \quad x \neq k .
$$

There are totally seven cases to discuss.

Case 1: $i \in N_{1}$. In this case, $b_{i}(x)=b_{i}^{\prime \prime}, a_{i j}(x)=a_{i j}^{\prime \prime}$. Since $u=f_{i}(-k-\varepsilon)$ and $u \leq f_{j}\left(\rho_{j}\right) \leq v$, owing to the definition of $\varepsilon$ and (5), it have

$$
\begin{gathered}
F_{i}(-1 / \varepsilon)=\frac{b_{i}^{\prime \prime}}{\varepsilon}+a_{i i}^{\prime \prime} u+\sum_{j=1, j \neq i}^{n} a_{i j}^{\prime \prime} f_{j}\left(\rho_{j}\right)+J_{i} \geq \frac{b_{i}^{\prime \prime}}{\varepsilon}-\sum_{j=1}^{n}\left|a_{i j}^{\prime \prime}\right| \mu-\left|J_{i}\right|>0, \\
F_{i}(-k-\varepsilon) \leq b_{i}^{\prime \prime} k+a_{i i}^{\prime \prime} u+b_{i}^{\prime \prime} \varepsilon+\sum_{j \neq i, j=1}^{n} \max \left\{a_{i j}^{\prime \prime} u, a_{i j}^{\prime \prime} v\right\}+J_{i} \leq 0 .
\end{gathered}
$$

Then, a $\bar{\rho}_{i} \in(-1 / \varepsilon,-k-\varepsilon]$ can be found such that $F_{i}\left(\bar{\rho}_{i}\right)=0$ due to the continuity of $F_{i}(x)$.

Case 2: $i \in N_{2}$. In this case, $b_{i}(x)=b_{i}^{\prime \prime}, a_{i j}(x)=a_{i j}^{\prime \prime}$. Since $f_{i}(-k)=u, f_{i}(-T)=f_{i}(T)$ and $-T<T$, from (5) and (6), we obtain

$$
\begin{gathered}
F_{i}(-k+\varepsilon) \leq b_{i}^{\prime \prime} k+a_{i i}^{\prime \prime} f_{i}(-k)+\left(a_{i i}^{\prime \prime} l-b_{i}^{\prime \prime}\right) \varepsilon+\sum_{j=1, j \neq i}^{n} \max \left\{a_{i j}^{\prime \prime} u, a_{i j}^{\prime \prime} v\right\}+J_{i} \leq 0, \\
F_{i}(-T-\varepsilon)>-b_{i}^{\prime \prime} T+a_{i i}^{\prime \prime} f_{i}(-T)+\left(-a_{i i}^{\prime \prime} l+b_{i}^{\prime \prime}\right) \varepsilon+\sum_{j \neq i, j=1}^{n} \min \left\{a_{i j}^{\prime \prime} u, a_{i j}^{\prime \prime} v\right\}+J_{i} \geq 0 .
\end{gathered}
$$

A $\bar{\rho}_{i} \in[-k+\varepsilon,-T-\varepsilon)$ can be found such that $F_{i}\left(\bar{\rho}_{i}\right)=0$.

Case 3: $i \in N_{3}$. In this case, $b_{i}(x)=b_{i}^{\prime}, a_{i j}(x)=a_{i j}^{\prime}$. Because $f_{i}(0)=c, f_{i}(-T)=f_{i}(T)$ and $-T<T$, from (7) and (8), we have

$$
\begin{gathered}
F_{i}(-T+\varepsilon)>-b_{i}^{\prime} T+a_{i i}^{\prime} f_{i}(-T)+\left(a_{i i}^{\prime} l-b_{i}^{\prime}\right) \varepsilon+\sum_{j \neq i, j=1}^{n} \min \left\{a_{i j}^{\prime} u, a_{i j}^{\prime} v\right\}+J_{i} \geq 0, \\
F_{i}(-\varepsilon) \leq a_{i i}^{\prime} c+\left(-a_{i i}^{\prime} l+b_{i}^{\prime}\right) \varepsilon+\sum_{j=1, j \neq i}^{n} \max \left\{a_{i j}^{\prime} u, a_{i j}^{\prime} v\right\}+J_{i} \leq 0 .
\end{gathered}
$$

So, there is a $\bar{\rho}_{i} \in(-T+\varepsilon,-\varepsilon]$ such that $F_{i}\left(\bar{\rho}_{i}\right)=0$.

Case 4: $i \in N_{4}$. In this case, $b_{i}(x)=b_{i}^{\prime}, a_{i j}(x)=a_{i j}^{\prime}$. Since $f_{i}(0)=c$, from (7) and (8), we get

$$
\begin{gathered}
F_{i}(\varepsilon) \leq a_{i i}^{\prime} c+\left(-a_{i i}^{\prime} l-b_{i}^{\prime}\right) \varepsilon+\sum_{j=1, j \neq i}^{n} \max \left\{a_{i j}^{\prime} u, a_{i j}^{\prime} v\right\}+J_{i} \leq 0, \\
F_{i}(T-\varepsilon) \geq-b_{i}^{\prime} T+a_{i i}^{\prime} f_{i}(T)+\left(a_{i i}^{\prime} l+b_{i}^{\prime}\right) \varepsilon+\sum_{j=1, j \neq i}^{n} \min \left\{a_{i j}^{\prime} u, a_{i j}^{\prime} v\right\}+J_{i} \geq 0 .
\end{gathered}
$$

Thus, there is a $\bar{\rho}_{i} \in[\varepsilon, T-\varepsilon]$ such that $F_{i}\left(\bar{\rho}_{i}\right)=0$.

Case 5: $i \in N_{5}$. In this case, $b_{i}(x)=b_{i}^{\prime \prime}, a_{i j}(x)=a_{i j}^{\prime \prime}$. Since $k>-k$ and $f_{i}(k)=u$, from (5) and (6), we have

$$
F_{i}(T+\varepsilon) \geq-b_{i}^{\prime \prime} T+a_{i i}^{\prime \prime} f_{i}(T)+\left(-a_{i i}^{\prime \prime} l-b_{i}^{\prime \prime}\right) \varepsilon+\sum_{j=1, j \neq i}^{n} \min \left\{a_{i j}^{\prime \prime} u, a_{i j}^{\prime \prime} v\right\}+J_{i} \geq 0
$$




$$
F_{i}(k-\varepsilon) \leq-b_{i}^{\prime \prime} k+a_{i i}^{\prime \prime} f_{i}(k)+\left(a_{i i}^{\prime \prime} l+b_{i}^{\prime \prime}\right) \varepsilon+\sum_{j=1, j \neq i}^{n} \max \left\{a_{i j}^{\prime \prime} u, a_{i j}^{\prime \prime} v\right\}+J_{i} \leq 0 .
$$

Similarly, there exists a $\bar{\rho}_{i} \in[T+\varepsilon, k-\varepsilon]$ such that $F_{i}\left(\bar{\rho}_{i}\right)=0$.

Case 6: $i \in N_{6}$. In this case, $b_{i}(x)=b_{i}^{\prime \prime}, a_{i j}(x)=a_{i j}^{\prime \prime}$. Since $k>-k$ and $f_{i}(k)=u$, from (5) and (9), we have

$$
\begin{aligned}
& F_{i}(k+\varepsilon) \leq-b_{i}^{\prime \prime} k+a_{i i}^{\prime \prime} f_{i}(k)+\left(a_{i i}^{\prime \prime} \bar{l}-b_{i}^{\prime \prime}\right) \varepsilon+\sum_{j=1, j \neq i}^{n} \max \left\{a_{i j}^{\prime \prime} u, a_{i j}^{\prime \prime} v\right\}+J_{i} \leq 0, \\
& F_{i}(d-\varepsilon) \geq-b_{i}^{\prime \prime} d+a_{i i}^{\prime \prime} f_{i}(d)+\left(-a_{i i}^{\prime \prime} \bar{l}+b_{i}^{\prime \prime}\right) \varepsilon+\sum_{j=1, j \neq i}^{n} \min \left\{a_{i j}^{\prime \prime} u, a_{i j}^{\prime \prime} v\right\}+J_{i} \geq 0 .
\end{aligned}
$$

Thus, there is a $\bar{\rho}_{i} \in[k+\varepsilon, d-\varepsilon]$ such that $F_{i}\left(\bar{\rho}_{i}\right)=0$.

Case 7: $i \in N_{7}$. In this case, $b_{i}(x)=b_{i}^{\prime \prime}, a_{i j}(x)=a_{i j}^{\prime \prime}$. Owing to $v=f_{i}(d+\varepsilon)$ and $u \leq f_{j}\left(\rho_{j}\right) \leq v$, from (9), we obtain

$$
\begin{gathered}
F_{i}(d+\varepsilon) \geq-b_{i}^{\prime \prime} d+a_{i i}^{\prime \prime} v-b_{i}^{\prime \prime} \varepsilon+\sum_{j=1, j \neq i}^{n} \min \left\{a_{i j}^{\prime \prime} u, a_{i j}^{\prime \prime} v\right\}+J_{i} \geq 0, \\
F_{i}(1 / \varepsilon)=-\frac{b_{i}^{\prime \prime}}{\varepsilon}+a_{i i}^{\prime \prime} d+\sum_{j=1, j \neq i}^{n} a_{i j}^{\prime \prime} f_{j}\left(\rho_{j}\right)+J_{i}<-\frac{b_{i}^{\prime \prime}}{\varepsilon}+\sum_{j=1}^{n}\left|a_{i j}^{\prime \prime}\right| \mu+\left|J_{i}\right|<0 .
\end{gathered}
$$

By the continuity of $F_{i}(x)$, there is a $\bar{\rho}_{i} \in[d+\varepsilon, 1 / \varepsilon)$ such that $F_{i}\left(\bar{\rho}_{i}\right)=0$.

Next, define a map $M: \widetilde{\Omega}_{\varepsilon} \rightarrow \widetilde{\Omega}_{\varepsilon}$ as $M\left(\rho_{1}, \rho_{2}, \ldots, \rho_{n}\right)=\left(\bar{\rho}_{1}, \bar{\rho}_{2}, \ldots, \bar{\rho}_{n}\right)$. Since the map $M$ is continuous, we can find a point $\rho^{*}=\left(\rho_{1}^{*}, \rho_{2}^{*}, \ldots, \rho_{n}^{*}\right)^{T} \in \widetilde{\Omega}_{\varepsilon}$ such that $M\left(\rho_{1}^{*}, \rho_{2}^{*}, \ldots, \rho_{n}^{*}\right)=\left(\rho_{1}^{*}, \rho_{2}^{*}, \ldots, \rho_{n}^{*}\right)$, which implies that the following condition is satisfied:

$$
-b_{i}\left(\rho_{i}^{*}\right) \rho_{i}^{*}+\sum_{j=1}^{n} a_{i j}\left(\rho_{i}^{*}\right) f_{j}\left(\rho_{j}^{*}\right)+J_{i}=0 .
$$

Hence, $\rho^{*}$ is an equilibrium point of $\operatorname{SSHNN}(2)$ in $\widetilde{\Omega}_{\varepsilon}$. Due to the arbitrariness of $\widetilde{\Omega}_{\varepsilon}$, we can get that there are at least $7^{n}$ equilibria located in $\Omega_{\varepsilon}$ for $\operatorname{SSHNN}(2)$.

\subsection{Stability of multiple equilibrium points}

In this section, we will study the stability of the multiple equilibria. First, the positive invariant set of SSHNN (2) need to be identified to analyze the stability further.

Denote a set $\Phi$ as

$$
\begin{gathered}
\Phi=\left\{\prod_{i=1}^{n}[-1 / \varepsilon,-k-\varepsilon]_{1}^{\delta_{1}^{(i)}} \times[-T+\varepsilon,-\varepsilon]_{2}^{\delta_{2}^{(i)}} \times[T+\varepsilon, k-\varepsilon]_{3}^{\delta_{3}^{(i)}} \times[d+\varepsilon, 1 / \varepsilon]_{4}^{\delta_{4}^{(i)}},\right. \\
\left.\left(\delta_{1}^{(i)}, \delta_{2}^{(i)}, \delta_{3}^{(i)}, \delta_{4}^{(i)}\right)=(1,0,0,0) \text { or }(0,1,0,0) \text { or }(0,0,1,0) \text { or }(0,0,0,1)\right\},
\end{gathered}
$$

and denote the set $\Psi=\Omega_{\varepsilon}-\Phi$. Then we can clearly obtain that there are $4^{n}$ regions in set $\Phi$ and $7^{n}-4^{n}$ regions in set $\Psi$.

Theorem 2 For SSHNN (2) with activation function (1), if conditions (5)-(9) hold for $i=1,2, \ldots, n$, then the set $\Phi$ is positively invariant. 
Proof: Arbitrarily pick a region

$$
\widetilde{\Phi}=\prod_{i \in N_{1}}[-1 / \varepsilon,-k-\varepsilon] \times \prod_{i \in N_{2}}[-T+\varepsilon,-\varepsilon] \times \prod_{i \in N_{3}}[T+\varepsilon, k-\varepsilon] \times \prod_{i \in N_{4}}[d+\varepsilon, 1 / \varepsilon] \subset \Phi,
$$

where $N_{1}, N_{2}, N_{3}$ and $N_{4}$ are subsets of $\{1,2, \ldots, n\}, N_{i} \cap N_{j}=\emptyset(i \neq j, i, j=1,2,3,4)$ and $N_{1} \cup N_{2} \cup$ $N_{3} \cup N_{4}=\{1,2, \ldots, n\}$. When the set $\widetilde{\Phi}$ is positively invariant, we can obtain that $\rho(t ; \rho(0)) \in \widetilde{\Phi}$ for all $t>0$ with any initial condition $\rho(0) \in \widetilde{\Phi}$. Assume that it is false, then four cases will be discussed in the following text.

Case 1: Suppose that there is a $i \in N_{1}$ and $t^{*}>0$ satisfying that $\rho_{i}\left(t^{*}\right)=-k-\varepsilon, \rho_{i}(t) \in[-1 / \varepsilon,-k-\varepsilon]$ and $\dot{\rho}_{i}\left(t^{*}\right)>0$ for all $0 \leq t \leq t^{*}$. Owing to (5), the definition of $f_{i}\left(\rho_{i}\right)$ and the enough small $\varepsilon$ we can obtain

$$
\begin{aligned}
\dot{\rho}_{i}\left(t^{*}\right) & =-b_{i}^{\prime \prime} \rho_{i}\left(t^{*}\right)+a_{i i}^{\prime \prime} f_{i}\left(\rho_{i}\left(t^{*}\right)\right)+\sum_{j=1, j \neq i}^{n} a_{i j}^{\prime \prime} f_{j}\left(\rho_{j}\left(t^{*}\right)\right)+J_{i} \\
& \leq b_{i}^{\prime \prime} k+a_{i i}^{\prime \prime} u+b_{i}^{\prime \prime} \varepsilon+\sum_{j=1, j \neq i}^{n} \max \left\{a_{i j}^{\prime \prime} u, a_{i j}^{\prime \prime} v\right\}+J_{i} \leq 0,
\end{aligned}
$$

which implies that it is a contradiction with $\dot{\rho}_{i}\left(t^{*}\right)>0$.

Case 2: Suppose that there is a $i \in N_{2}$ and $t^{*}>0$ satisfying that $\rho_{i}\left(t^{*}\right)=-T+\varepsilon, \dot{\rho}_{i}\left(t^{*}\right)<0$ and $\rho_{i}(t) \in[-T+\varepsilon,-\varepsilon]$ for all $0 \leq t \leq t^{*}$, or $\rho_{i}\left(t^{*}\right)=-\varepsilon, \dot{\rho}_{i}\left(t^{*}\right)>0$ and $\rho_{i}(t) \in[-T+\varepsilon,-\varepsilon]$ for all $0 \leq t \leq t^{*}$. For the former, owing to Proposition $1,(7)$, the definition of $f_{i}\left(\rho_{i}\right)$ and the enough small $\varepsilon$ we can obtain

$$
\begin{aligned}
\dot{\rho}_{i}\left(t^{*}\right) & =-b_{i}^{\prime} \rho_{i}\left(t^{*}\right)+a_{i i}^{\prime} f_{i}\left(\rho_{i}\left(t^{*}\right)\right)+\sum_{j=1, j \neq i}^{n} a_{i j}^{\prime} f_{j}\left(\rho_{j}\left(t^{*}\right)\right)+J_{i} \\
& \geq-b_{i}^{\prime} T+a_{i i}^{\prime} f_{i}(-T)-\left(b_{i}^{\prime}-a_{i i}^{\prime} l\right) \varepsilon+\sum_{j=1, j \neq i}^{n} \min \left\{a_{i j}^{\prime} u, a_{i j}^{\prime} v\right\}+J_{i} \geq 0,
\end{aligned}
$$

which implies that it is a contradiction with $\dot{\rho}_{i}\left(t^{*}\right)<0$. In addition, for the second one, it can be obtained with the similar method.

Case 3: Suppose that there is a $i \in N_{3}$ and $t^{*}>0$ satisfying that $\rho_{i}\left(t^{*}\right)=T+\varepsilon, \dot{\rho}_{i}\left(t^{*}\right)<0$ and $\rho_{i}(t) \in[T+\varepsilon, k-\varepsilon]$ for all $0 \leq t \leq t^{*}$, or $\rho_{i}\left(t^{*}\right)=k-\varepsilon, \dot{\rho}_{i}\left(t^{*}\right)>0$ and $\rho_{i}(t) \in[T+\varepsilon, k-\varepsilon]$ for all $0 \leq t \leq t^{*}$. For the former, owing to Proposition 1, (6), the definition of $f_{i}\left(\rho_{i}\right)$ and the enough small $\varepsilon$ we can obtain

$$
\begin{aligned}
\dot{\rho}_{i}\left(t^{*}\right) & =-b_{i}^{\prime \prime} \rho_{i}\left(t^{*}\right)+a_{i i}^{\prime \prime} f_{i}\left(\rho_{i}\left(t^{*}\right)\right)+\sum_{j=1, j \neq i}^{n} a_{i j}^{\prime \prime} f_{j}\left(\rho_{j}\left(t^{*}\right)\right)+J_{i} \\
& >-b_{i}^{\prime \prime} T+a_{i i}^{\prime \prime} f_{i}(T)-\left(b_{i}^{\prime \prime}+a_{i i}^{\prime \prime} l\right) \varepsilon+\sum_{j=1, j \neq i}^{n} \min \left\{a_{i j}^{\prime \prime} u, a_{i j}^{\prime \prime} v\right\}+J_{i} \geq 0,
\end{aligned}
$$

which implies that it is a contradiction with $\dot{\rho}_{i}\left(t^{*}\right)<0$. Then the second one can be proved with the similar method.

Case 4: Suppose that there is a $i \in N_{4}$ and $t^{*}>0$ satisfying that $\rho_{i}\left(t^{*}\right)=d+\varepsilon, \dot{\rho}_{i}\left(t^{*}\right) \leq 0$ and $\rho_{i}(t) \in[d+\varepsilon, 1 / \varepsilon]$ for all $0 \leq t \leq t^{*}$. Owing to $(9)$, the definition of $f_{i}\left(\rho_{i}\right)$ and the enough small $\varepsilon$, we can obtain

$$
\begin{aligned}
\dot{\rho}_{i}\left(t^{*}\right) & =-b_{i}^{\prime \prime} \rho_{i}\left(t^{*}\right)+a_{i i}^{\prime \prime} f_{i}\left(\rho_{i}\left(t^{*}\right)\right)+\sum_{j=1, j \neq i}^{n} a_{i j}^{\prime \prime} f_{j}\left(\rho_{j}\left(t^{*}\right)\right)+J_{i} \\
& \geq-b_{i}^{\prime \prime} d+a_{i i}^{\prime \prime} v-b_{i}^{\prime \prime} \varepsilon+\sum_{j=1, j \neq i}^{n} \min \left\{a_{i j}^{\prime \prime} u, a_{i j}^{\prime \prime} v\right\}+J_{i}>0,
\end{aligned}
$$


which implies that it is a contradiction with $\dot{\rho}_{i}\left(t^{*}\right) \leq 0$.

Based on the above discussion we get the conclusion that the set $\Phi$ is positively invariant. This completes the proof.

Then, the stability of every equilibrium point of SSHNN (2) will be studied.

Theorem 3 For SSHNN (2) with activation function (1), if conditions (5)-(9) and the following conditions hold:

$$
\sum_{j \neq i, j=1}^{n}\left|a_{i j}^{\prime}\right| \max \{l, \bar{l}\}<\min \left\{b_{i}^{\prime},\left|a_{i i}^{\prime} l-b_{i}^{\prime}\right|,\left|a_{i i}^{\prime} l+b_{i}^{\prime}\right|,\left|a_{i i}^{\prime} \bar{l}-b_{i}^{\prime}\right|,\left|a_{i i}^{\prime} \bar{l}+b_{i}^{\prime}\right|\right\}
$$

and

$$
\sum_{j \neq i, j=1}^{n}\left|a_{i j}^{\prime \prime}\right| \max \{l, \bar{l}\}<\min \left\{b_{i}^{\prime \prime},\left|a_{i i}^{\prime \prime} l-b_{i}^{\prime \prime}\right|,\left|a_{i i}^{\prime \prime} l+b_{i}^{\prime \prime}\right|,\left|a_{i i}^{\prime \prime} \bar{l}-b_{i}^{\prime \prime}\right|,\left|a_{i i}^{\prime \prime} \bar{l}+b_{i}^{\prime \prime}\right|\right\},
$$

then SSHNN (2) can have $7^{n}$ equilibrium points, of which $4^{n}$ equilibrium points are locally exponentially stable (LES) and the other $7^{n}-4^{n}$ ones are unstable.

Proof: Here, we have the following two cases to discuss.

Case 1: When the equilibrium point is located in subset $\Phi$. Arbitrarily pick a subset

$$
\widetilde{\Phi}=\prod_{i \in N_{1}}[-1 / \varepsilon,-k-\varepsilon] \times \prod_{i \in N_{2}}[-T+\varepsilon,-\varepsilon] \times \prod_{i \in N_{3}}[T+\varepsilon, k-\varepsilon] \times \prod_{i \in N_{4}}[d+\varepsilon, 1 / \varepsilon] \subset \Phi,
$$

where $N_{1}, N_{2}, N_{3}$ and $N_{4}$ are subsets of $\{1,2, \ldots, n\}, N_{i} \cap N_{j}=\emptyset(i, j=1,2,3,4, i \neq j)$ and $N_{1} \cup N_{2} \cup$ $N_{3} \cup N_{4}=\{1,2, \ldots, n\}$.

Denote $\rho^{*}$ as an equilibrium point located in $\widetilde{\Phi}$ and denote $\rho(t ; \rho(0))$ as the solution of Eq.(2), where $\rho(0) \in \widetilde{\Phi}$ is the initial condition. Next, make a transformation $\gamma(t)=\rho(t ; \rho(0))-\rho^{*}$ and we can obtain that $\rho(t ; \rho(0)) \in \widetilde{\Phi}$ for all $t \geq 0$ because the set $\widetilde{\Phi}$ is positively invariant. When $\rho(t ; \rho(0)) \in \Phi, \sum_{j \in N_{1}} f_{j}\left(\rho_{j}\right)$ and $\sum_{j \in N_{4}} f_{j}\left(\rho_{j}\right)$ are constants. That is, $\sum_{j \in N_{1}} f_{j}\left(\rho_{j}\right)=\sum_{j \in N_{1}} f_{j}\left(\rho_{j}^{*}\right)$ and $\sum_{j \in N_{4}} f_{j}\left(\rho_{j}\right)=\sum_{j \in N_{4}} f_{j}\left(\rho_{j}^{*}\right)$. Hence, we can obtain that:

$$
\dot{\gamma}_{i}(t)=-b_{i}\left(\rho_{i}\right) \gamma_{i}(t)+\sum_{j \in N_{2}} a_{i j}\left(\rho_{i}\right)\left[f_{j}\left(\rho_{j}^{*}+\gamma_{j}(t)\right)-f_{j}\left(\rho_{j}^{*}\right)\right]+\sum_{j \in N_{3}} a_{i j}\left(\rho_{i}\right)\left[f_{j}\left(\rho_{j}^{*}+\gamma_{j}(t)\right)-f_{j}\left(\rho_{j}^{*}\right)\right] .
$$

Without loss of generality, assume that $N_{2}=\{1,2, \ldots, s\}, N_{3}=\{s+1, s+2, \ldots, w\}, s \leq w \leq n$ and Eq. (17) can be rewritten as

$$
\dot{\gamma}_{i}(t)=-b_{i}\left(\rho_{i}\right) \gamma_{i}(t)+\sum_{j=1}^{s} a_{i j}\left(\rho_{i}\right) l \gamma_{j}(t)-\sum_{j=s+1}^{w} a_{i j}\left(\rho_{i}\right) l \gamma_{j}(t) .
$$

Hence, we can obtain the coefficient matrix of $\widehat{A}_{1}$ in system (18), which is shown as follows:

$$
\widehat{A}_{1}=\left(\begin{array}{ccccccccc}
-b_{1}^{\prime}+a_{11}^{\prime} l & \cdots & a_{1 s}^{\prime} l & -a_{1(s+1)}^{\prime} l & \cdots & -a_{1 w}^{\prime} l & 0 & \cdots & 0 \\
\ldots & \cdots & \cdots & \cdots & \cdots & \cdots & \cdots & \cdots & \cdots \\
a_{s 1}^{\prime} l & \cdots & -b_{s}^{\prime}+a_{s s}^{\prime} l & -a_{s(s+1)}^{\prime} l & \cdots & -a_{s w}^{\prime} l & 0 & \cdots & 0 \\
a_{(s+1) 1}^{\prime \prime} l & \cdots & a_{(s+1) s}^{\prime \prime} l & -b_{s+1}^{\prime \prime}-a_{(s+1)(s+1)}^{\prime \prime} & \cdots & -a_{(s+1) w}^{\prime \prime} l & 0 & \cdots & 0 \\
\cdots & \cdots & \cdots & \cdots & \cdots & \cdots & \cdots & \cdots & \cdots \\
a_{w 1}^{\prime \prime} l & \cdots & a_{w s}^{\prime \prime} l & -a_{w(s+1)}^{\prime \prime} l & \cdots & -b_{w}^{\prime \prime}-a_{w w}^{\prime \prime} l & 0 & \cdots & 0 \\
a_{(w+1) 1}^{\prime \prime} l & \cdots & a_{(w+1) s}^{\prime \prime} l & -a_{(w+1)(s+1)}^{\prime \prime} l & \cdots & -a_{(w+1) w}^{\prime \prime} l & -b_{w+1}^{\prime \prime} & \cdots & 0 \\
\cdots & \cdots & \cdots & \cdots & \cdots & \cdots & \cdots & \cdots & \cdots \\
a_{n 1}^{\prime \prime} l & \cdots & a_{n s}^{\prime \prime} l & -a_{n(s+1)}^{\prime \prime} l & \cdots & -a_{n w}^{\prime \prime} l & 0 & \cdots & -b_{n}^{\prime \prime}
\end{array}\right) .
$$


If conditions (15) and (16) are satisfied, then the matrix $\widehat{A}_{1}$ is a SDDM. According to Proposition 1 and Lemma 1 , there are $n$ eigenvalues with negative real part in matrix $\widehat{A}_{1}$, which implies that $\rho^{*}$ is a LES equilibrium point. Therefore, we can obtain that in each subset of $\Phi$, there exists a stable equilibrium point because we arbitrarily choose $\widetilde{\Phi}$ in $\Phi$. Hence, SSHNN (2) has $4^{n}$ LES equilibrium points in Case 1.

Case 2: When the equilibrium point is located in subset $\Psi$. Arbitrarily pick a subset

$$
\begin{aligned}
\widetilde{\Psi}= & \prod_{i \in N_{1}}[-1 / \varepsilon,-k-\varepsilon] \times \prod_{i \in N_{2}}[-k+\varepsilon,-T-\varepsilon] \times \prod_{i \in N_{3}}[-T+\varepsilon,-\varepsilon] \times \prod_{i \in N_{4}}[\varepsilon, T-\varepsilon] \\
& \times \prod_{i \in N_{5}}[T+\varepsilon, k-\varepsilon] \times \prod_{i \in N_{6}}[k+\varepsilon, d-\varepsilon] \times \prod_{i \in N_{7}}[d+\varepsilon, 1 / \varepsilon] \subset \Psi,
\end{aligned}
$$

where $N_{i}(i=1,2, \ldots, 7)$ are subsets of $\{1,2, \ldots, n\}, N_{1} \cup N_{2} \cup N_{3} \cup N_{4} \cup N_{5} \cup N_{6} \cup N_{7}=\{1,2, \ldots, n\}, N_{i} \cap$ $N_{j}=\emptyset(i \neq j)$ and $N_{2} \cup N_{4} \cup N_{6} \neq \emptyset$. Using the similar way as in Case 1, let $N_{2}=\{1,2, \ldots, p\}, N_{4}=$ $\{p+1, p+2, \ldots, q\}, N_{6}=\{q+1, q+2, \ldots, r\}, N_{3}=\{r+1, r+2, \ldots, s\}$ and $N_{5}=\{s+1, s+2, \ldots, w\}$. Make a assumption that $\rho(t ; \rho(0))$ is a solution of $\operatorname{SSHNN}(2)$ being close to $\rho^{*}$, according to the definition of instability, if the solution goes out of $\widetilde{\Psi}$, then we can get that the equilibrium point $\rho^{*}$ is unstable. Then, suppose that $\rho(t ; \rho(0)) \in \widetilde{\Psi}$ for all $t \geq 0$, let $\gamma(t)=\rho(t ; \rho(0))-\rho^{*}$ and we obtain that:

$$
\begin{aligned}
\dot{\gamma}_{i}(t)= & -b_{i}\left(\rho_{i}\right) \gamma_{i}(t)+\sum_{j=1}^{p} a_{i j}\left(\rho_{i}\right) l \gamma_{j}(t)-\sum_{j=p+1}^{q} a_{i j}\left(\rho_{i}\right) l \gamma_{j}(t) \\
& +\sum_{j=q+1}^{r} a_{i j}\left(\rho_{i}\right) \bar{l} \gamma_{j}(t)+\sum_{j=r+1}^{s} a_{i j}\left(\rho_{i}\right) l \gamma_{j}(t)-\sum_{j=s+1}^{w} a_{i j}\left(\rho_{i}\right) l \gamma_{j}(t) .
\end{aligned}
$$

Hence, we can obtain the coefficient matrix of $\widehat{A}_{2}$ in system (19), which is shown as follows:

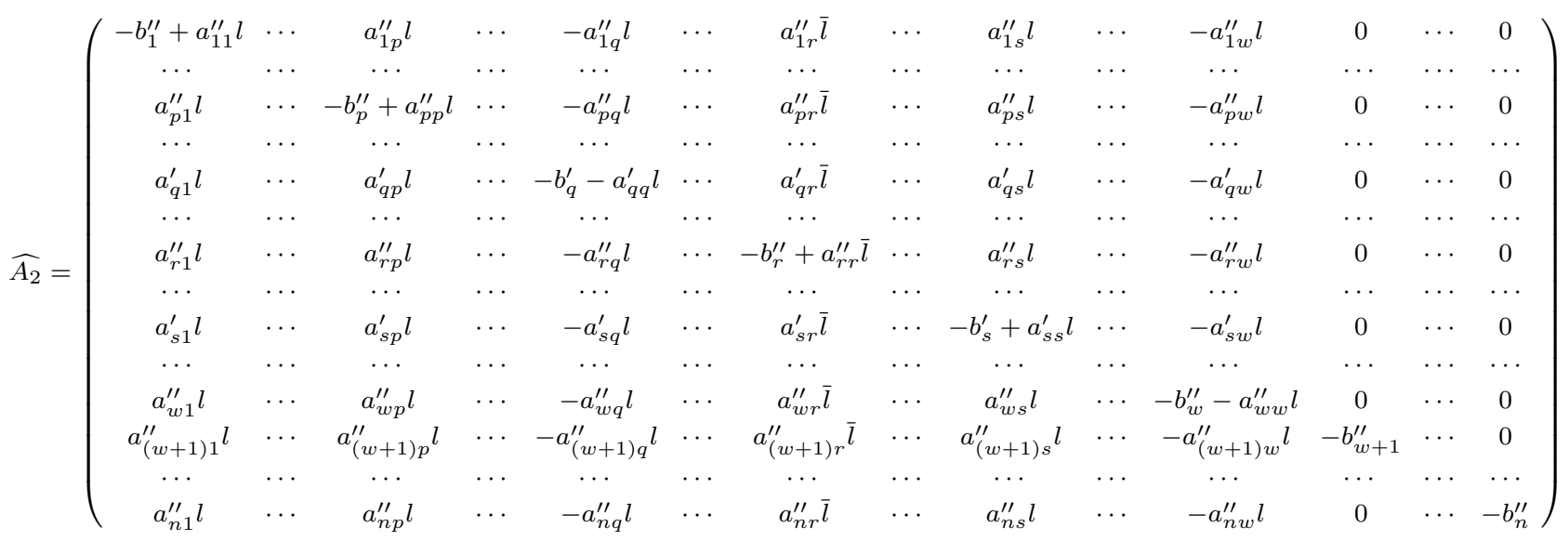

If conditions (15) and (16) are satisfied, then the matrix $\widehat{A}_{2}$ is a SDDM. According to Proposition 1 and Lemma 1 , there are $r$ with positive real part eigenvalues and $n-r$ with negative real part eigenvalues in matrix $\widehat{A}_{2}$, which implies that the equilibrium point $\rho^{*}$ is unstable. Therefore, we can obtain that in each subset of $\Psi$, there exists an unstable equilibrium point because we arbitrarily choose $\widetilde{\Psi}$ in $\Psi$. Hence, in Case 2, SSHNN (2) has $7^{n}-4^{n}$ unstable equilibrium points.

According to Cases 1-2, we get the conclusion that $\operatorname{SSHNN}(2)$ has at least $7^{n}$ equilibrium points, of which $4^{n}$ equilibrium points are LES and the other $7^{n}-4^{n}$ ones are unstable.

Remark 1 In [36], multistability of competitive neural networks (CNNs) with the Gaussian-wavelet-type activation functions was studied, in which it had been proven that CNNs can have $5^{n}$ equilibria and $3^{n}$ of them are LS. Compared with CNNs, SSHNNs could possess more total equilibria and more LS equilibria 
with the same activation function. Besides, in [35], multistability of SSHNNs with the Mexican-hat-type activation function was analyzed. Compared with the above activation functions, it have been shown in this paper HNNs under state-dependent switching with the Gaussian-wavelet-type activation functions could have more stable equilibria. These two cases both suggest that the storage capacity of the neural network is improved. So we can get the conclusion that both state-dependent switched NNs model and Gaussianwavelet-type activation functions can improve the storage capacity of NNs.

Remark 2 When $0<T<k$, the LES equilibria are not only located in the saturated regions $(-\infty,-k)$ and $(k,+\infty)$, but also located in the unsaturated regions $(-T, 0)$ and $(T, k)$. From Theorem 2 and Theorem 3, we can get that $\rho(t ; \rho(0))$ will converge to one of stable equilibrium in the $4^{n}$ regions of $\Phi$ when the initial condition $\rho(0)$ is located in $\Phi$. Therefore, we can derive that $\Phi$ is the basin of attraction in system (2) and it probably can be enlarged, which is a difficult work we will concentrate on in the future.

After discussing the coexistence and stability of multiple equilibrium points in the case of $0<T<k$, we will study the other two cases (i.e., $k<T<d$ and $T>d$ ) in the following text.

If the switching threshold $T$ satisfies that $k<T<d$, the following theorem is given.

Theorem 4 For SSHNN (2) with activation function (1), if the conditions (15)-(16) and the following conditions hold:

$$
\begin{gathered}
-b_{i}^{\prime} k+a_{i i}^{\prime} u+\sum_{j \neq i, j=1}^{n} \min \left\{a_{i j}^{\prime} u, a_{i j}^{\prime} v\right\}+J_{i}>0, \\
-b_{i}^{\prime \prime} T+a_{i i}^{\prime \prime} f_{i}(T)+\sum_{j \neq i, j=1}^{n} \max \left\{a_{i j}^{\prime \prime} u, a_{i j}^{\prime \prime} v\right\}+J_{i}<0, \\
-b_{i}^{\prime} T+a_{i i}^{\prime} f_{i}(T)+\sum_{j \neq i, j=1}^{n} \max \left\{a_{i j}^{\prime} u, a_{i j}^{\prime} v\right\}+J_{i}<0, \\
a_{i i}^{\prime} c+\sum_{j \neq i, j=1}^{n} \max _{i j}\left\{a_{i j}^{\prime} u, a_{i j}^{\prime} v\right\}+J_{i}<0, \\
-b_{i}^{\prime \prime} d+a_{i i}^{\prime \prime} v+\sum_{j \neq i, j=1}^{n} \min \left\{a_{i j}^{\prime \prime} u, a_{i j}^{\prime \prime} v\right\}+J_{i}>0, \\
b_{i}^{\prime \prime} T+a_{i i}^{\prime \prime} u+\sum_{j \neq i, j=1}^{n} \max \left\{a_{i j}^{\prime \prime} u, a_{i j}^{\prime \prime} v\right\}+J_{i}<0,
\end{gathered}
$$

then SSHNN (2) has $6^{n}$ equilibrium points, of which $4^{n}$ equilibrium points are LES and the other $6^{n}-4^{n}$ ones are unstable. The positively invariant set of SSHNN (2) is

$$
\begin{gathered}
\Phi_{1}=\left\{\prod_{i=1}^{n}(-\infty,-T)^{\delta_{1}^{(i)}} \times(-k, 0)^{\delta_{2}^{(i)}} \times(k, T)^{\delta_{3}^{(i)}} \times(d,+\infty)^{\delta_{4}^{(i)}},\left(\delta_{1}^{(i)}, \delta_{2}^{(i)},\right.\right. \\
\left.\left.\delta_{3}^{(i)}, \delta_{4}^{(i)}\right)=(1,0,0,0) \text { or }(0,1,0,0) \text { or }(0,0,1,0) \text { or }(0,0,0,1)\right\} .
\end{gathered}
$$

Proof: The proof of Theorem 4 is similar to that of Theorem 1-3 in the case $0<T<k$, so it is omitted here. We only need to give the reason why the number of total equilibria is $6^{n}$ instead of $7^{n}$. If $\rho_{i}(t)$ is located in $(-T,-k)$, from $(20)$ we can get that

$$
\dot{\rho}_{i}(t) \geq b_{i}^{\prime} k+a_{i i}^{\prime} u+\sum_{j=1, j \neq i}^{n} \min \left\{a_{i j}^{\prime} u, a_{i j}^{\prime} v\right\}+J_{i}>-b_{i}^{\prime} k+a_{i i}^{\prime} u+\sum_{j=1, j \neq i}^{n} \min \left\{a_{i j}^{\prime} u, a_{i j}^{\prime} v\right\}+J_{i}>0 .
$$


So, for any initial condition $\rho_{i}(0) \in(-T,-k), \rho_{i}(t)$ will enter the region $(-k, 0)$ as time goes on and there is no equilibrium point in $(-T,-k)$, this completes the proof.

In addition, if the switching threshold $T$ satisfies $T>d$, the following theorem is given.

Theorem 5 For SSHNN (2) with activation function (1), If conditions (15)-(16) and the following conditions hold:

$$
\begin{array}{r}
b_{i}^{\prime} k+a_{i i}^{\prime} u+\sum_{j \neq i, j=1}^{n} \max \left\{a_{i j}^{\prime} u, a_{i j}^{\prime} v\right\}+J_{i}<0, \\
-b_{i}^{\prime \prime} T+a_{i i}^{\prime \prime} v+\sum_{j \neq i, j=1}^{n} \min \left\{a_{i j}^{\prime \prime} u, a_{i j}^{\prime \prime} v\right\}+J_{i}>0, \\
-b_{i}^{\prime} T+a_{i i}^{\prime} v+\sum_{j \neq i, j=1}^{n} \max \left\{a_{i j}^{\prime} u, a_{i j}^{\prime} v\right\}+J_{i}<0, \\
a_{i i}^{\prime} c+\sum_{j \neq i, j=1}^{n} \min \left\{a_{i j}^{\prime} u, a_{i j}^{\prime} v\right\}+J_{i}>0, \\
-b_{i}^{\prime} d+a_{i i}^{\prime} v+\sum_{j \neq i, j=1}^{n} \min \left\{a_{i j}^{\prime} u, a_{i j}^{\prime} v\right\}+J_{i}>0, \\
b_{i}^{\prime \prime} T+a_{i i}^{\prime \prime} u+\sum_{j \neq i, j=1}^{n} \max \left\{a_{i j}^{\prime \prime} u, a_{i j}^{\prime \prime} v\right\}+J_{i}<0, \\
b_{i}^{\prime} T+a_{i i}^{\prime} u+\sum_{j \neq i, j=1}^{n} \min \left\{a_{i j}^{\prime} u, a_{i j}^{\prime} v\right\}+J_{i}>0,
\end{array}
$$

then SSHNN (2) has $7^{n}$ equilibrium points, of which $5^{n}$ equilibrium points are LES and the other $7^{n}-5^{n}$ ones are unstable. The positively invariant set of SSHNN (2) is

$$
\begin{gathered}
\Phi_{2}=\left\{\prod_{i=1}^{n}(-\infty,-T)^{\delta_{1}^{(i)}} \times(-T,-k)^{\delta_{2}^{(i)}} \times(0, k)^{\delta_{3}^{(i)}} \times(d, T)^{\delta_{4}^{(i)}} \times(T,+\infty)^{\delta_{5}^{(i)}},\left(\delta_{1}^{(i)}, \delta_{2}^{(i)}, \delta_{3}^{(i)}, \delta_{4}^{(i)},\right.\right. \\
\left.\left.\delta_{5}^{(i)}\right)=(1,0,0,0,0) \text { or }(0,1,0,0,0) \text { or }(0,0,1,0,0) \text { or }(0,0,0,1,0) \text { or }(0,0,0,0,1)\right\} .
\end{gathered}
$$

Proof: Here the proof of Theorem 5 is omitted because it is similar to that of Theorem 1-3.

Remark 3 In these three cases, there are different number of total equilibria and stable equilibria in SSHNNs (2), which depends on where the switching threshold $T$ is located. Compared with the case $0<$ $T<k$ and $T>d$, there are less total equilibria in the case $k<T<d$. Compared with the case $0<T<k$

and $k<T<d$, there are more LS equilibria in the case $T>d$. If SSHNNs with this type of activation function can be used in associative memory in practice, we can make SSHNNs storage more memory patterns by adjusting the switching threshold $T \in(d,+\infty)$.

\section{$4 \quad$ Numerical example}

In this section, three examples will be given to verify the correctness of the theoretical results.

Example 1 First, when $T \in(0,4)$, we consider a kind of 2-neuron SSHNN:

$$
\left\{\begin{array}{l}
\dot{\rho}_{1}(t)=-b_{1}\left(\rho_{1}(t)\right) \rho_{1}(t)+a_{11}\left(\rho_{1}(t)\right) f\left(\rho_{1}(t)\right)+a_{12}\left(\rho_{1}(t)\right) f\left(\rho_{2}(t)\right)+J_{1} \\
\dot{\rho}_{2}(t)=-b_{2}\left(\rho_{2}(t)\right) \rho_{2}(t)+a_{21}\left(\rho_{2}(t)\right) f\left(\rho_{1}(t)\right)+a_{22}\left(\rho_{2}(t)\right) f\left(\rho_{2}(t)\right)+J_{2}
\end{array}\right.
$$


where $J_{1}=2, J_{2}=3$, the switching threshold $T=2$ and

$$
\begin{aligned}
& b_{1}\left(\rho_{1}(t)\right)=\left\{\begin{array}{ll}
0.50, & \left|\rho_{1}(t)\right|>2 \\
0.80, & \left|\rho_{1}(t)\right| \leq 2
\end{array} \quad b_{2}\left(\rho_{2}(t)\right)= \begin{cases}0.70, & \left|\rho_{2}(t)\right|>2 \\
1.20, & \left|\rho_{2}(t)\right| \leq 2\end{cases} \right. \\
& a_{11}\left(\rho_{1}(t)\right)=\left\{\begin{array}{ll}
3.00, & \left|\rho_{1}(t)\right|>2 \\
-2.00, & \left|\rho_{1}(t)\right| \leq 2
\end{array} \quad a_{12}\left(\rho_{1}(t)\right)= \begin{cases}0.10, & \left|\rho_{1}(t)\right|>2 \\
-0.10, & \left|\rho_{1}(t)\right| \leq 2\end{cases} \right. \\
& a_{21}\left(\rho_{2}(t)\right)=\left\{\begin{array}{ll}
0.10, & \left|\rho_{2}(t)\right|>2 \\
-0.10, & \left|\rho_{2}(t)\right| \leq 2
\end{array} \quad a_{22}\left(\rho_{2}(t)\right)= \begin{cases}5.00, & \left|\rho_{2}(t)\right|>2 \\
-3.00, & \left|\rho_{2}(t)\right| \leq 2\end{cases} \right.
\end{aligned}
$$

The activation function is defined by:

$$
f(\rho)=\left\{\begin{array}{lc}
-2, & -\infty<\rho<-4 \\
\rho+2, & -4 \leq \rho<0 \\
-\rho+2, & 0 \leq \rho \leq 4 \\
\rho-6, & 4<\rho \leq 8 \\
2, & 8<\rho<+\infty .
\end{array}\right.
$$

It is clear that SSHNN (33) satisfies the conditions which are proposed in Theorem 1-3, so we can derive that SSHNN (33) with activation function (34) has $7^{2}=49$ equilibria, among which $4^{2}=16$ equilibria are LES and the other 33 ones are unstable. Fig. 2 depicts the relationship between $\rho_{1}$ and $\rho_{2}$ in system (33) with 500 random initial values $\rho(0)=\left(\rho_{1}(0), \rho_{2}(0)\right)^{T}$ starting from the region $[-10,15] \times[-10,15]$. We can see 16 stable equilibrium points clearly from Fig. 2. In addition, Figs. 3-4 display the time response of $\rho_{1}$ and $\rho_{2}$ of system (33) and it can be seen that as time goes on, $\rho_{1}(t)$ and $\rho_{2}(t)$ converge to 16 values. From Figs. 2-4 we can see that the obtained results are testified.

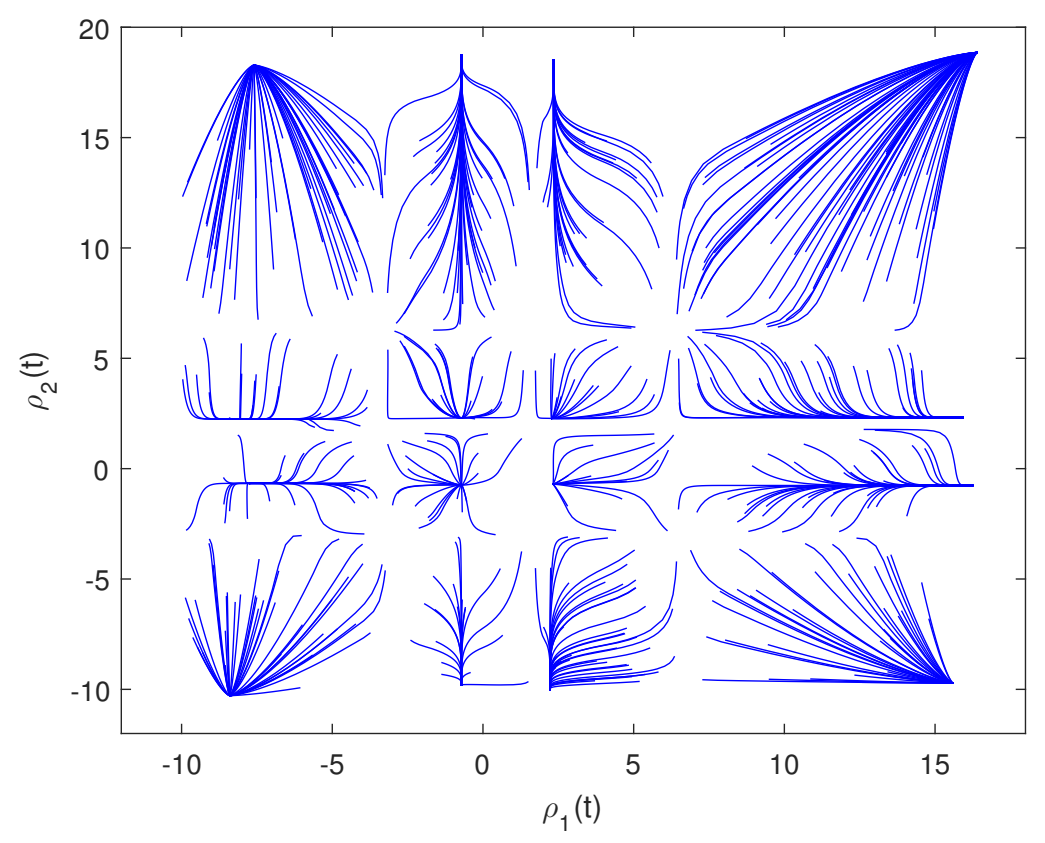

Fig. 2. Phase diagram of system (33) with 500 random initial conditions $\rho(0)=\left(\rho_{1}(0), \rho_{2}(0)\right)^{T}$ starting from the region $[-10,15] \times[-10,15]$ in Example 1. 


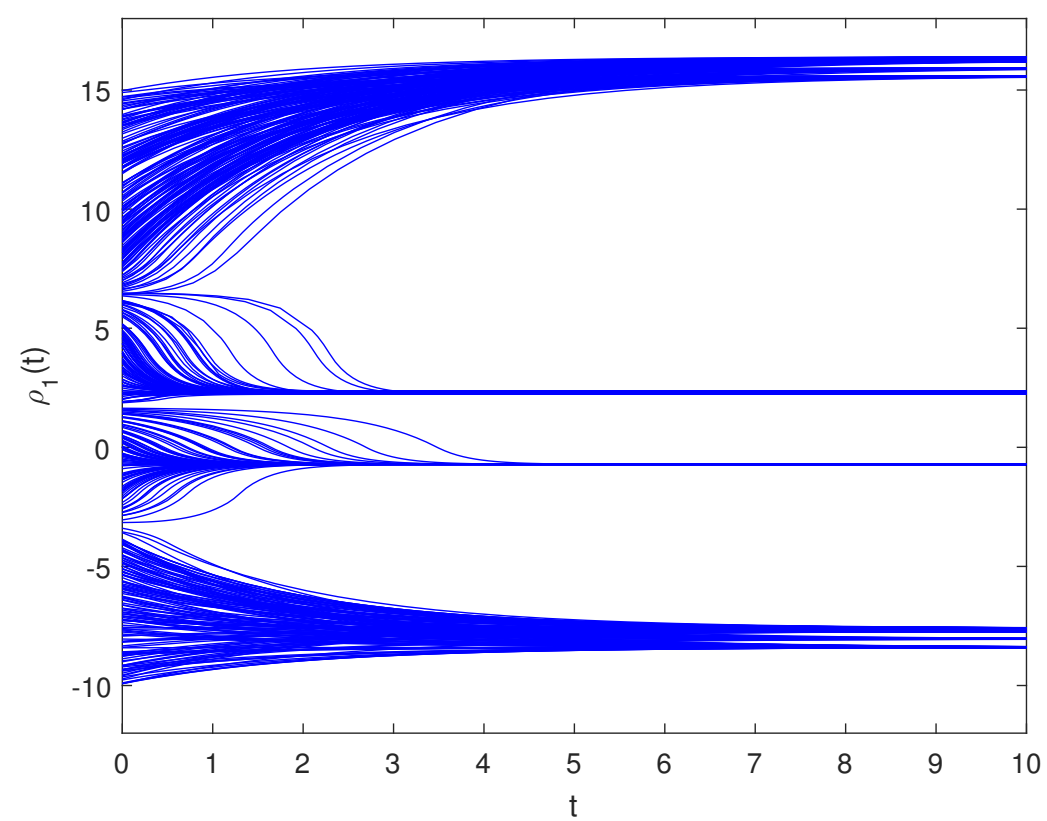

Fig. 3. The time response of $\rho_{1}$ of system (33) with 500 random initial conditions $\rho(0)=\left(\rho_{1}(0), \rho_{2}(0)\right)^{T} \in[-10,15] \times[-10,15]$ in Example 1.

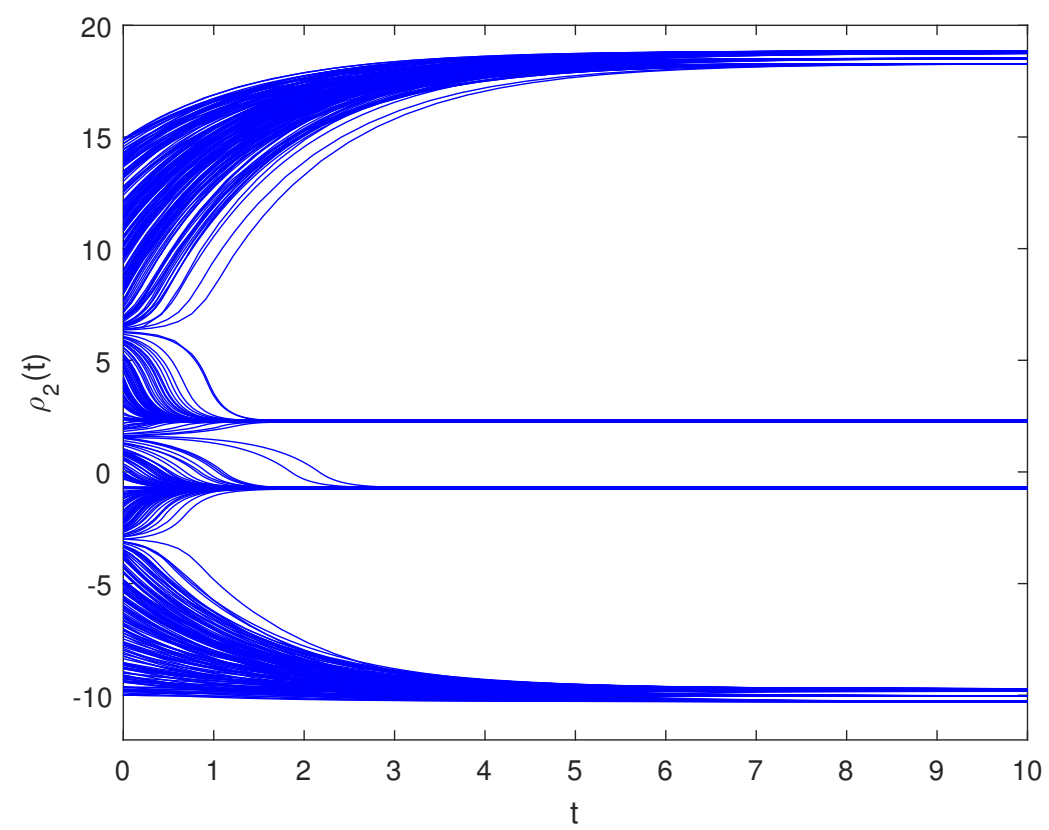

Fig. 4. The time response of $\rho_{2}$ of system (33) with 500 random initial conditions $\rho(0)=\left(\rho_{1}(0), \rho_{2}(0)\right)^{T} \in[-10,15] \times[-10,15]$ in Example 1.

Example 2 Then, when $T \in(4,8)$, we consider a 2-neuron SSHNN:

$$
\left\{\begin{array}{l}
\dot{\rho}_{1}(t)=-b_{1}\left(\rho_{1}(t)\right) \rho_{1}(t)+a_{11}\left(\rho_{1}(t)\right) f\left(\rho_{1}(t)\right)+a_{12}\left(\rho_{1}(t)\right) f\left(\rho_{2}(t)\right)+J_{1} \\
\dot{\rho}_{2}(t)=-b_{2}\left(\rho_{2}(t)\right) \rho_{2}(t)+a_{21}\left(\rho_{2}(t)\right) f\left(\rho_{1}(t)\right)+a_{22}\left(\rho_{2}(t)\right) f\left(\rho_{2}(t)\right)+J_{2}
\end{array}\right.
$$

where $J_{1}=2, J_{2}=3$, the switching threshold $T=6$ and

$$
b_{1}\left(\rho_{1}(t)\right)=\left\{\begin{array}{ll}
0.50, & \left|\rho_{1}(t)\right|>6 \\
1.00, & \left|\rho_{1}(t)\right| \leq 6
\end{array} \quad b_{2}\left(\rho_{2}(t)\right)= \begin{cases}0.70, & \left|\rho_{2}(t)\right|>6 \\
1.20, & \left|\rho_{2}(t)\right| \leq 6\end{cases}\right.
$$




$$
\begin{aligned}
& a_{11}\left(\rho_{1}(t)\right)=\left\{\begin{array}{ll}
3.50, & \left|\rho_{1}(t)\right|>6 \\
-2.00, & \left|\rho_{1}(t)\right| \leq 6
\end{array} \quad a_{12}\left(\rho_{1}(t)\right)= \begin{cases}0.10, & \left|\rho_{1}(t)\right|>6 \\
-0.10, & \left|\rho_{1}(t)\right| \leq 6\end{cases} \right. \\
& a_{21}\left(\rho_{2}(t)\right)=\left\{\begin{array}{ll}
0.10, & \left|\rho_{2}(t)\right|>6 \\
-0.10, & \left|\rho_{2}(t)\right| \leq 6
\end{array} \quad a_{22}\left(\rho_{2}(t)\right)= \begin{cases}4.00, & \left|\rho_{2}(t)\right|>6 \\
-3.00, & \left|\rho_{2}(t)\right| \leq 6\end{cases} \right.
\end{aligned}
$$

The activation function is (34), which is the same as in Example 1.

It is clear that SSHNN (35) satisfies the conditions which are proposed in Theorem 4, so we can get that SSHNN (35) with activation function (34) has $6^{2}=36$ equilibria, among which $4^{2}=16$ equilibria are LES and the other 20 ones are unstable. Fig. 5 depicts the relationship between $\rho_{1}$ and $\rho_{2}$ in system (35) with 500 random initial values $\rho(0)=\left(\rho_{1}(0), \rho_{2}(0)\right)^{T}$ starting from the region $[-10,15] \times[-10,15]$. We can see 16 stable equilibrium points clearly from Fig. 5 . We can also see that for any initial condition $\rho_{i}(0) \in(-6,-4), \rho_{i}(t)$ will enter the region $(-4,0)$ as time goes on for $i=1,2$ from Fig. 5. In addition, Figs. 6-7 display the time response of $\rho_{1}$ and $\rho_{2}$ of system (35) and it can be seen that as time goes on, $\rho_{1}(t)$ and $\rho_{2}(t)$ converge to 16 values. From Figs. 5-7 we can see that the obtained results are testified.

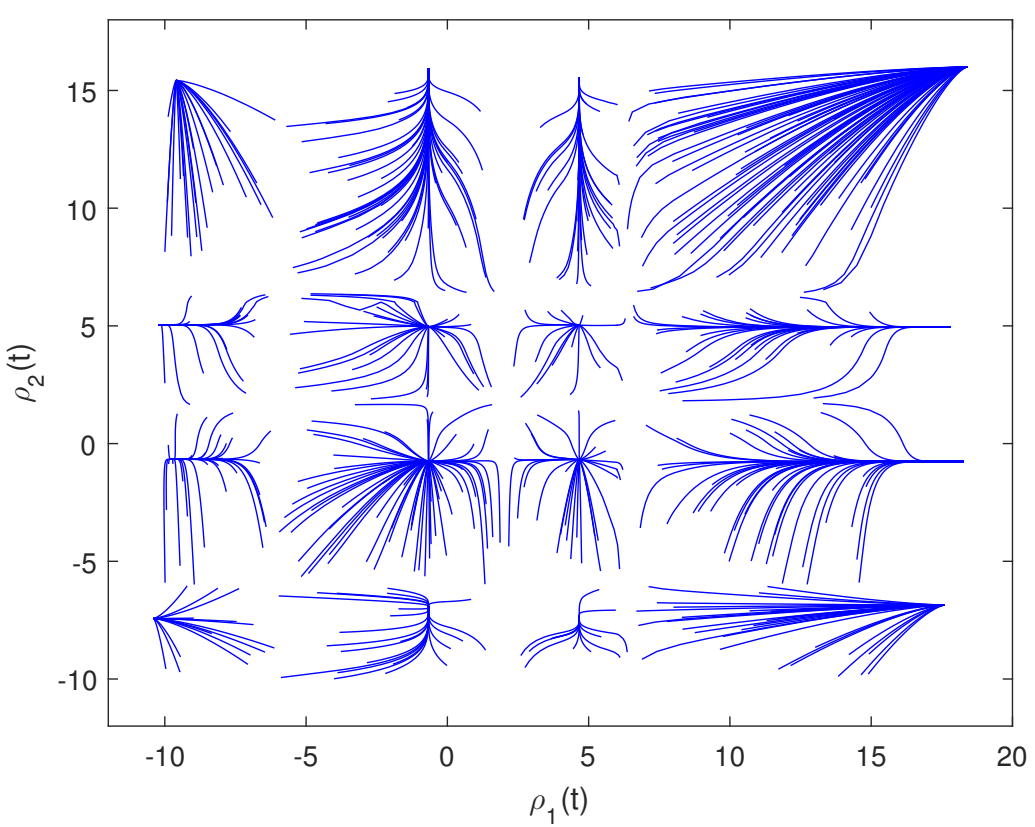

Fig. 5. Phase diagram of system (35) with 500 random initial conditions $\rho(0)=\left(\rho_{1}(0), \rho_{2}(0)\right)^{T}$ starting from the region $[-10,15] \times[-10,15]$ in Example 2. 




Fig. 6. The time response of $\rho_{1}$ of system (35) with 500 random initial conditions $\rho(0)=\left(\rho_{1}(0), \rho_{2}(0)\right)^{T} \in[-10,15] \times[-10,15]$ in Example 2.

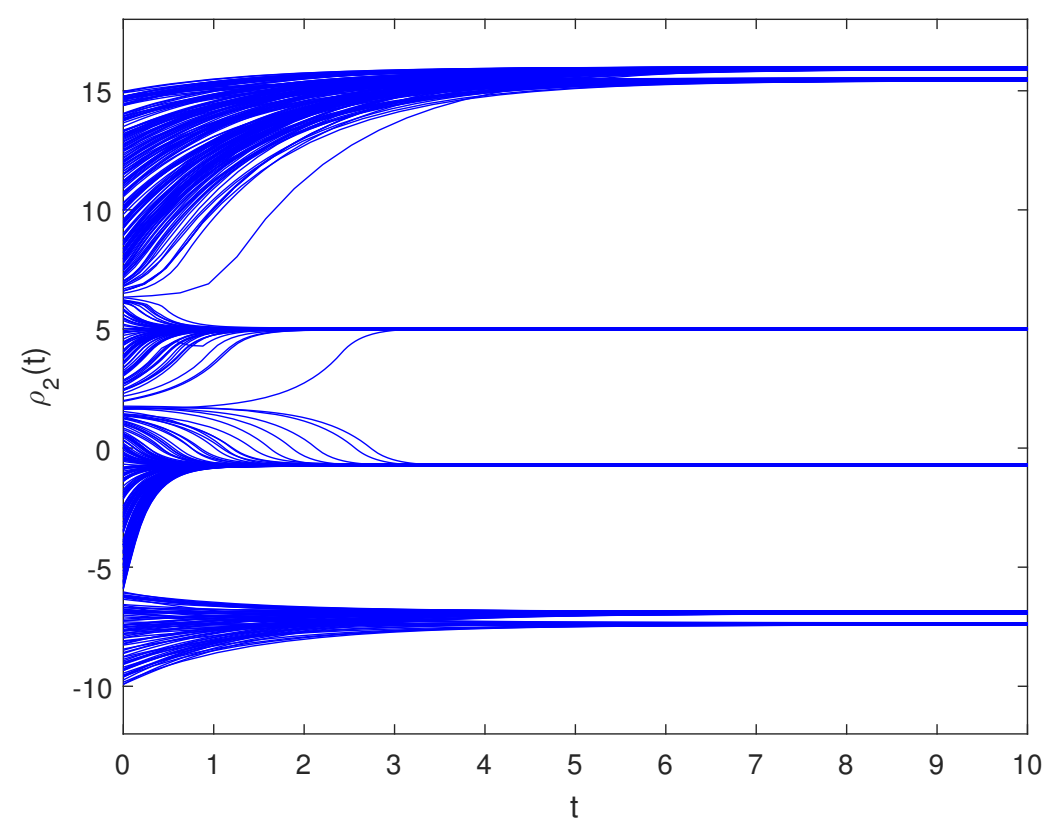

Fig. 7. The time response of $\rho_{2}$ of system (35) with 500 random initial conditions

$$
\rho(0)=\left(\rho_{1}(0), \rho_{2}(0)\right)^{T} \in[-10,15] \times[-10,15] \text { in Example } 2 .
$$

Example 3 Finally, when $T \in(8,+\infty)$, we consider a 2-neuron SSHNN:

$$
\left\{\begin{array}{l}
\dot{\rho}_{1}(t)=-b_{1}\left(\rho_{1}(t)\right) \rho_{1}(t)+a_{11}\left(\rho_{1}(t)\right) f\left(\rho_{1}(t)\right)+a_{12}\left(\rho_{1}(t)\right) f\left(\rho_{2}(t)\right)+J_{1} \\
\dot{\rho}_{2}(t)=-b_{2}\left(\rho_{2}(t)\right) \rho_{2}(t)+a_{21}\left(\rho_{2}(t)\right) f\left(\rho_{1}(t)\right)+a_{22}\left(\rho_{2}(t)\right) f\left(\rho_{2}(t)\right)+J_{2}
\end{array}\right.
$$

where $J_{1}=2, J_{2}=3$, the switching threshold $T=10$ and

$$
b_{1}\left(\rho_{1}(t)\right)=\left\{\begin{array}{ll}
0.50, & \left|\rho_{1}(t)\right|>10 \\
1.00, & \left|\rho_{1}(t)\right| \leq 10
\end{array} \quad b_{2}\left(\rho_{2}(t)\right)= \begin{cases}0.60, & \left|\rho_{2}(t)\right|>10 \\
1.15, & \left|\rho_{2}(t)\right| \leq 10\end{cases}\right.
$$




$$
\begin{aligned}
& a_{11}\left(\rho_{1}(t)\right)=\left\{\begin{array}{ll}
4.00, & \left|\rho_{1}(t)\right|>10 \\
3.50, & \left|\rho_{1}(t)\right| \leq 10
\end{array} \quad a_{12}\left(\rho_{1}(t)\right)= \begin{cases}0.10, & \left|\rho_{1}(t)\right|>10 \\
-0.10, & \left|\rho_{1}(t)\right| \leq 10\end{cases} \right. \\
& a_{21}\left(\rho_{2}(t)\right)=\left\{\begin{array}{ll}
0.10, & \left|\rho_{2}(t)\right|>10 \\
-0.10, & \left|\rho_{2}(t)\right| \leq 10
\end{array} \quad a_{22}\left(\rho_{2}(t)\right)= \begin{cases}5.00, & \left|\rho_{2}(t)\right|>10 \\
4.00, & \left|\rho_{2}(t)\right| \leq 10\end{cases} \right.
\end{aligned}
$$

The activation function is (34), which is the same as in Example 1.

It is clear that SSHNN (36) satisfies the conditions which are proposed in Theorem 5, so we can get that SSHNN (36) with activation function (34) has $7^{2}=49$ equilibria, among which $5^{2}=25$ equilibria are LES and the other 24 ones are unstable. Fig. 8 depicts the relationship between $\rho_{1}$ and $\rho_{2}$ in system (36) with 800 random initial values $\rho(0)=\left(\rho_{1}(0), \rho_{2}(0)\right)^{T}$ starting from the region $[-16,22] \times[-16,22]$. We can see 25 stable equilibrium points clearly from Fig. 8. In addition, Figs. 9-10 display the time response of $\rho_{1}$ and $\rho_{2}$ of system (36) and it can be seen that as time goes on, $\rho_{1}(t)$ and $\rho_{2}(t)$ converge to 25 values. From Figs. 8-10 we can see that the obtained results are testified.

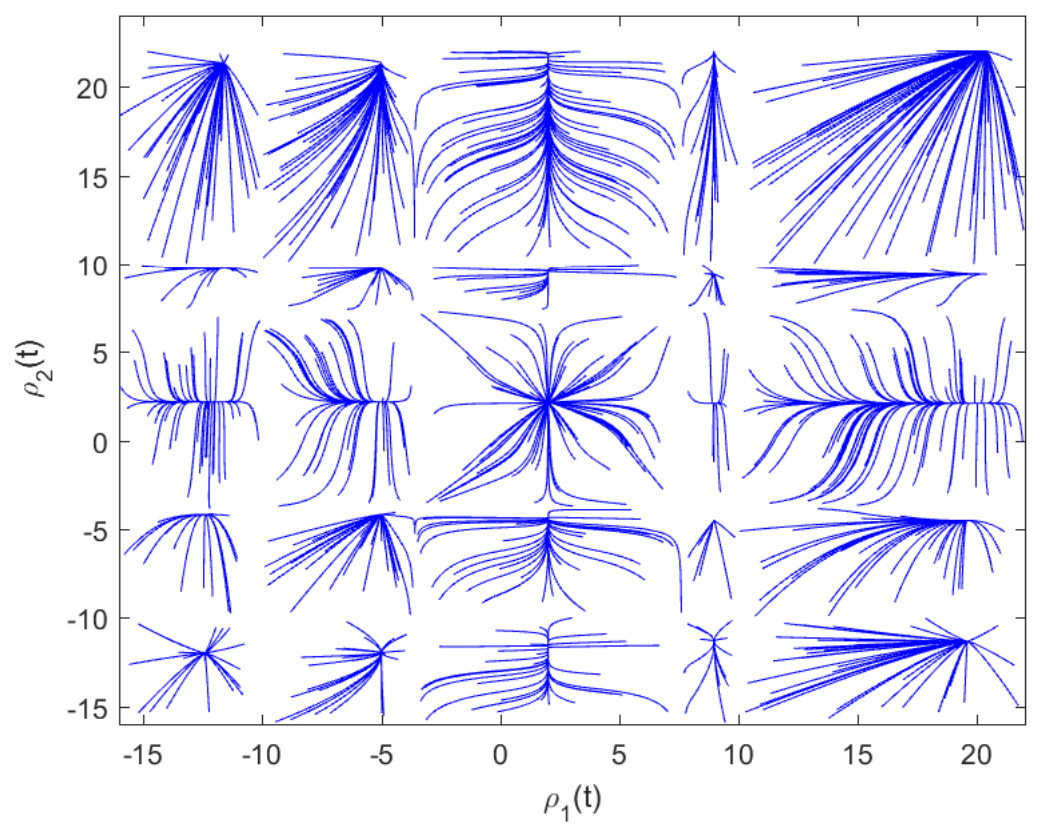

Fig. 8. Phase diagram of system (36) with 800 random initial conditions $\rho(0)=\left(\rho_{1}(0), \rho_{2}(0)\right)^{T}$ starting from the region $[-16,22] \times[-16,22]$ in Example 3. 


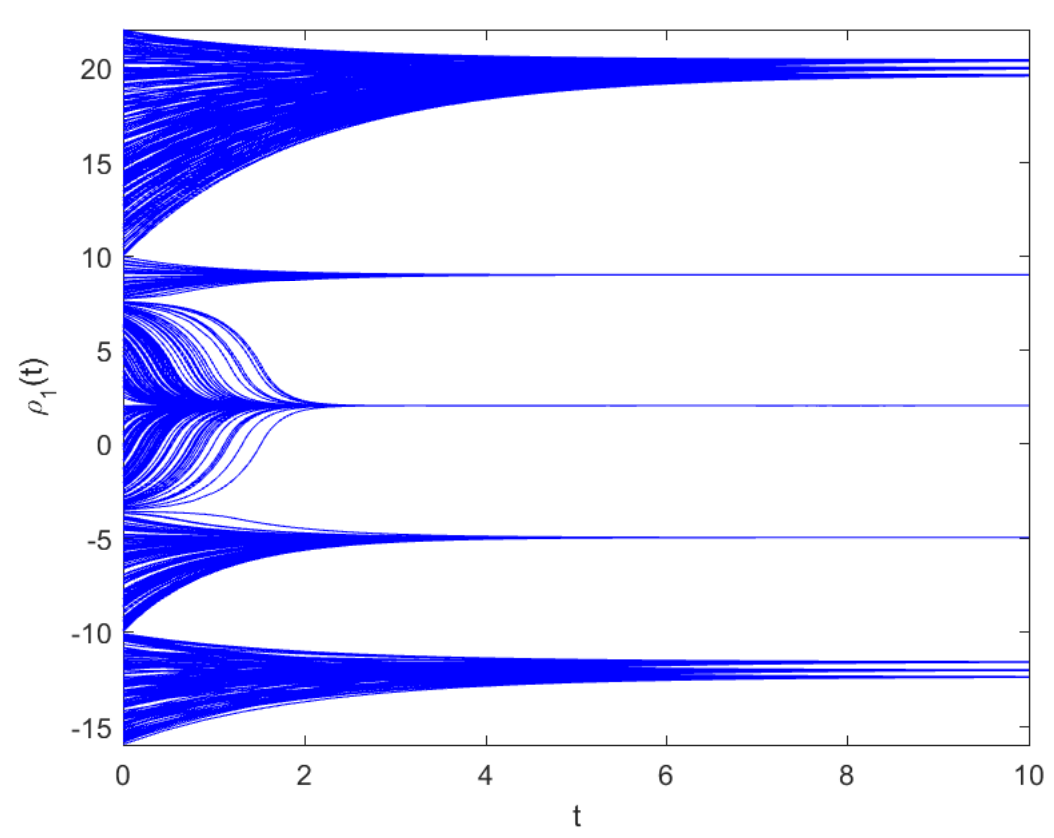

Fig. 9. The time response of $\rho_{1}$ of system (36) with 800 random initial conditions $\rho(0)=\left(\rho_{1}(0), \rho_{2}(0)\right)^{T} \in[-16,22] \times[-16,22]$ in Example 3.

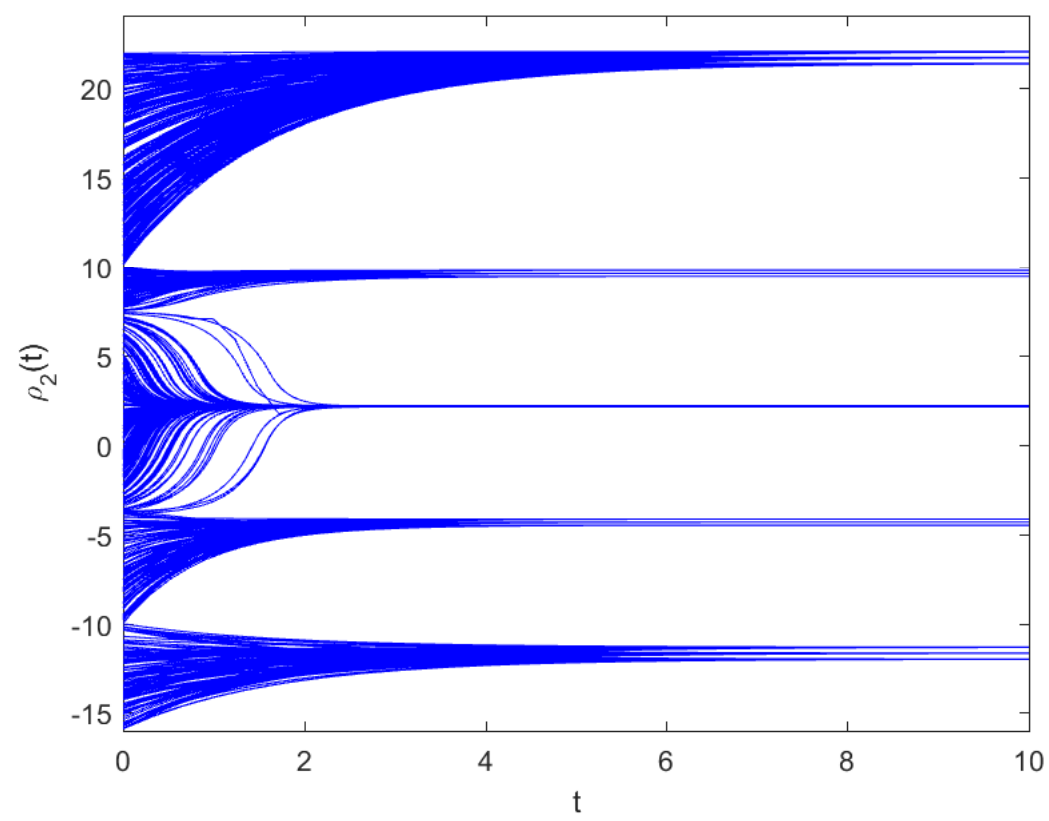

Fig. 10. The time response of $\rho_{2}$ of system (36) with 800 random initial conditions $\rho(0)=\left(\rho_{1}(0), \rho_{2}(0)\right)^{T} \in[-16,22] \times[-16,22]$ in Example 3.

Remark 4 In Example 1-3, we use different systems with the same activation function (34) because it can make the obtained results convincing. Compared with Example 1 and Example 2, there are 25 LES equilibria in Example 3, the number of LES equilibria is largest, which implies that if these three systems are used in associative memory application, system (36) in Example 3 can storage more memory patterns than system (33) and system (35) in Example 1-2. 


\section{Conclusions}

In this paper, the coexistence and multistability of equilibria of SSHNNs with Gaussian-wavelet-type activation function are proved. It has been shown that under some conditions, $n$-neuron SSHNNs can have $7^{n}$ or $6^{n}$ equilibria in total, of which $4^{n}$ or $5^{n}$ equilibria are LES. The number of total equilibria or LS equilibria depends on where the switching threshold is located. Compared with the neural networks without state-dependent switching, SSHNNs could possess more LS equilibria, which implies that the storage capacity is increased correspondingly. Finally, three examples are given to verify the correctness of the theoretical results. Moreover, the multistability of SSHNNs with delays has not been addressed which may have even richer dynamics than SSHNNs without delays, so it is a significant work to study the multistability of this model with some specified activation functions.

\section{Data availability}

Data sharing not applicable to this article as no datasets were generated or analysed during the current study.

\section{Conflict of Interest}

The authors declare that they have no conflict of interest.

\section{References}

[1] Cohen, M. A. and Grossberg, S. (1983). "Absolute stability of global pattern formation and parallel memory storage by competitive neural networks." IEEE Transactions on Systems, Man, and Cybernetics, 13(5), 815-826.

[2] Murino, V. (1998). "Structured neural networks for pattern recognition." IEEE Transactions on Systems, Man, and Cybernetics, Part B: Cybernetics, 28(4), 553-561.

[3] Cao, J. and Li, R. (2017). "Fixed-time synchronization of delayed memristor-based recurrent neural networks." Science China Information Sciences, 60(3), 032201.

[4] Zeng, Z., Huang, D. S. and Wang, Z. (2005). "Memory pattern analysis of cellular neural networks." Physics Letters A, 342(1-2), 114-128.

[5] Morita, M. (1993). "Associative memory with nonmonotone dynamics." Neural Networks, 6(1), 115126.

[6] Melin, P., Mendoza, O. and Castillo, O. (2011). "Face recognition with an improved interval type-2 fuzzy logic sugeno integral and modular neural networks." IEEE Transactions on Systems, Man, and Cybernetics-Part A: Systems and Humans, 41(5), 1001-1012.

[7] Huang, Z., Song, Q. and Feng, C. (2009). "Multistability in networks with self-excitation and highorder synaptic connectivity." IEEE Transactions on Circuits and Systems I: Regular Papers, 57(8), 2144-2155. 
[8] Nie, X., Liang, J. and Cao, J. (2019). "Multistability analysis of competitive neural networks with Gaussian-wavelet-type activation functions and unbounded time-varying delays." Applied Mathematics and Computation, 356, 449-468.

[9] Wang, L., Lu, W. and Chen, T. (2009). "Multistability of neural networks with a class of activation functions." International Symposium on Neural Networks, Shanghai, May 26-29, 323-332.

[10] Nie, X. and Zheng, W. X. (2015). "Multistability of neural networks with discontinuous non-monotonic piecewise linear activation functions and time-varying delays." Neural Networks, 65, 65-79.

[11] Nie, X. and Zheng, W. X. (2015). "Dynamical behaviors of multiple equilibria in competitive neural networks with discontinuous nonmonotonic piecewise linear activation functions." IEEE Transactions on Cybernetics, 46(3), 679-693.

[12] Huang, Y., Wang, Z., Zhang, H. and Zhang, T. (2010). "Multistability and enlarged basins of attraction in bidirectional associative memory neural networks." 2010 International Conference on Networking, Sensing and Control (ICNSC), Shenyang, April 10-12, 376-381.

[13] Lu, W. and Chen, T. (2005). "Dynamical behaviors of Cohen-Grossberg neural networks with discontinuous activation functions." Neural Networks, 18(3), 231-242.

[14] Chen, W. H., Luo, S. and Lu, X. (2015). "Multistability in a class of stochastic delayed Hopfield neural networks." Neural Networks, 68, 52-61.

[15] Kaslik, E. and Sivasundaram, S. (2011). "Multistability in impulsive hybrid Hopfield neural networks with distributed delays." Nonlinear Analysis: Real World Applications, 12(3), 1640-1649.

[16] Kaslik, E. and Sivasundaram, S. (2011). "Multiple periodic solutions in impulsive hybrid neural networks with delays." Applied Mathematics and Computation, 217(10), 4890-4899.

[17] Kaslik, E. and Sivasundaram, S. (2011). "Impulsive hybrid discrete-time Hopfield neural networks with delays and multistability analysis." Neural Networks, 24(4), 370-377.

[18] Wan, P., Sun, D., Zhao, M., Wan, L. and Jin, S. (2020). "Multistability and attraction basins of discrete-time neural networks with nonmonotonic piecewise linear activation functions." Neural Networks, 122, 231-238.

[19] Huang, Y. and Zhang, X. (2011). "Multistability properties of linear threshold discrete-time recurrent neural networks." International Journal of Information and Systems Sciences, 7(1), 1-10.

[20] Yi, Z. and Tan, K. K. (2004). "Multistability of discrete-time recurrent neural networks with unsaturating piecewise linear activation functions." IEEE Transactions on Neural Networks, 15(2), 329-336.

[21] Huang, Z., Wang, X. and Feng, C. (2010). "Multiperiodicity of periodically oscillated discrete-time neural networks with transient excitatory self-connections and sigmoidal nonlinearities." IEEE Transactions on Neural Networks, 21(10), 1643-1655. Networks, 21(10), 1643-1655.

[22] Zeng, Z. and Wang, J. (2006). "Multiperiodicity of discrete-time delayed neural networks evoked by periodic external inputs." IEEE Transactions on Neural Networks, 17(5), 1141-1151. 
[23] Cheng, C. Y., Lin, K. H. and Shih, C. W. (2006). "Multistability in recurrent neural networks." SIAM Journal on Applied Mathematics, 66(4), 1301-1320.

[24] Zeng, Z., Wang, J. and Liao, X. (2004). "Stability analysis of delayed cellular neural networks described using cloning templates." IEEE Transactions on Circuits and Systems I: Regular Papers, 51(11), 2313-2324.

[25] Wang, L. and Chen, T. (2012). "Multistability of neural networks with Mexican-hat-type activation functions." IEEE Transactions on Neural Networks and Learning Systems, 23(11), 1816-1826.

[26] Nie, X. and Zheng, W. X. (2015). "Multistability and instability of neural networks with discontinuous nonmonotonic piecewise linear activation functions." IEEE Transactions on Neural Networks and Learning Systems, 26(11), 2901-2913.

[27] Liu, P., Nie, X., Liang, J. and Cao, J. (2018). "Multiple Mittag-Leffler stability of fractional-order competitive neural networks with Gaussian activation functions." Neural Networks, 108, 452-465.

[28] Huang, G. and Cao, J. (2008). "Multistability of neural networks with discontinuous activation function." Communications in Nonlinear Science and Numerical Simulation, 13(10), 2279-2289.

[29] Zeng, Z., Huang, T. and Zheng, W. X. (2010). "Multistability of recurrent neural networks with timevarying delays and the piecewise linear activation function." IEEE Transactions on Neural Networks, $21(8), 1371-1377$.

[30] Zeng, Z. and Zheng, W. X. (2013). "Multistability of two kinds of recurrent neural networks with activation functions symmetrical about the origin on the phase plane." IEEE Transactions on Neural Networks and Learning Systems, 24(11), 1749-1762.

[31] Liu, P., Zeng, Z. and Wang, J. (2016). "Multistability of recurrent neural networks with nonmonotonic activation functions and mixed time delays." IEEE Transactions on Systems Man and CyberneticsSystems, 46(4), 512-523.

[32] Zeng, Z. and Zheng, W. X. (2012). "Multistability of neural networks with time-varying delays and concave-convex characteristics." IEEE Transactions on Neural Networks and Learning Systems, 23(2), 293-305.

[33] Guo, Z., Ou, S. and Wang, J. (2020). "Multistability of switched neural networks with sigmoidal activation functions under state-dependent switching." Neural Networks, 122, 239-252.

[34] Guo, Z., Liu, L. and Wang, J. (2018). "Multistability of switched neural networks with piecewise linear activation functions under state-dependent switching." IEEE Transactions on Neural Networks and Learning Systems, 30(7), 2052-2066.

[35] Guo, Z., Liu, L. and Wang, J. (2018). "Multistability for recurrent neural networks with piecewiselinear radial basis functions and state-dependent switching parameters." IEEE Transactions on Systems, Man, and Cybernetics: Systems, 50(11), 4458-4471.

[36] Nie, X., Cao, J. and Fei, S. (2019). "Multistability and instability of competitive neural networks with non-monotonic piecewise linear activation functions." Nonlinear Analysis: Real World Applications, 45, 799-821. 
[37] Tong, W. T. (1977). "Distribution of eigenvalues of a class of matrices." Acta Mathematica Sinica, 20(4), 272-275. 